دراسة بعض التغيرات النوعية في لحوم أصناف من الاجاج المحلي والمستورد المخزن بالتجميد

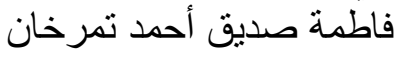

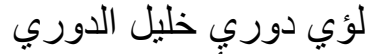

قسم علوم الأغذية و التقانات الإحيائية / كلية الزراعة و الغابات / جامعة الموصل ـ العراق

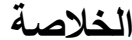

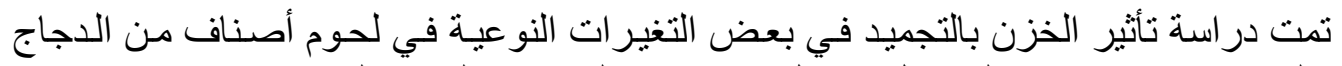

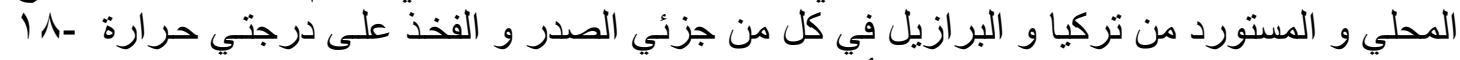

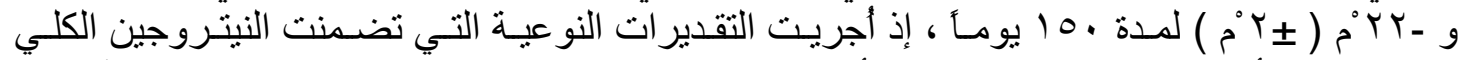

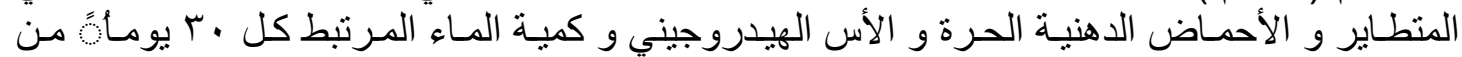

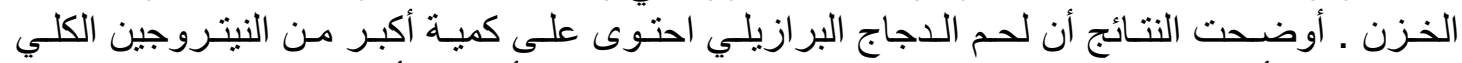

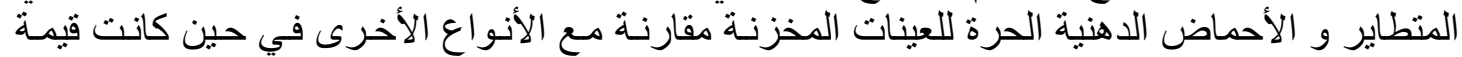

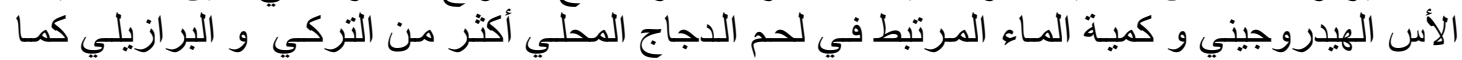

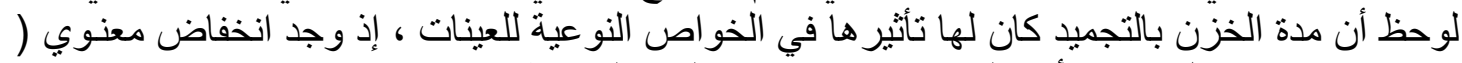

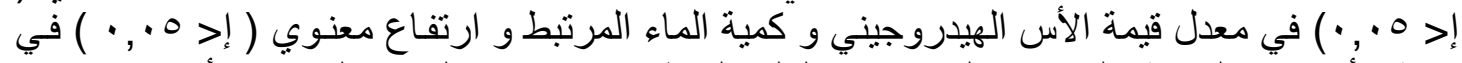

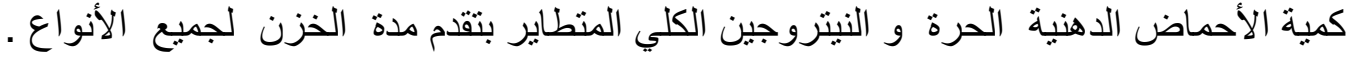

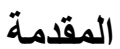

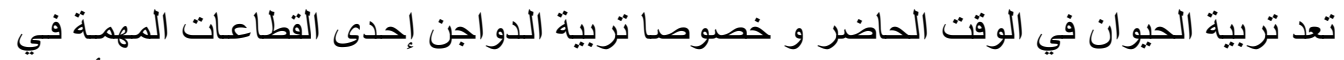

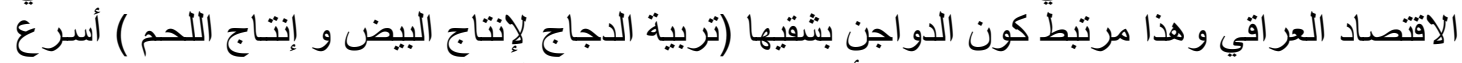

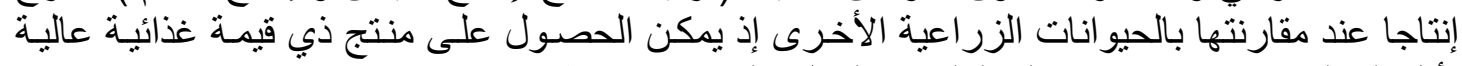

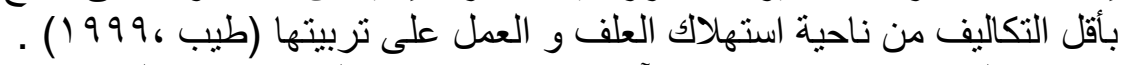

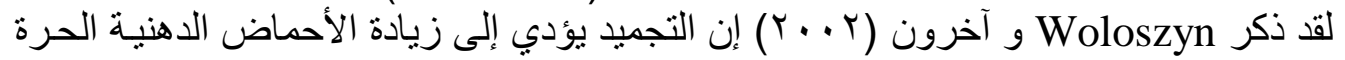

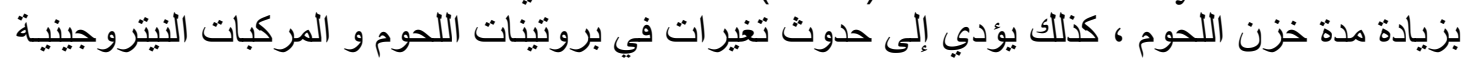

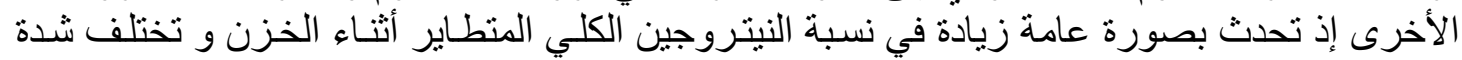

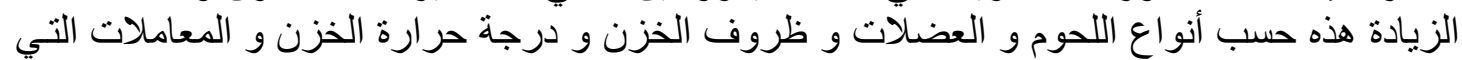

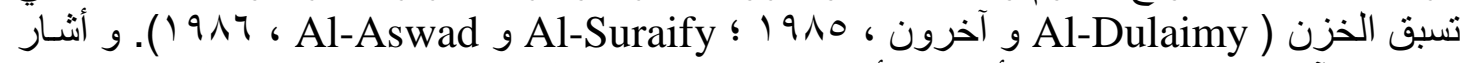

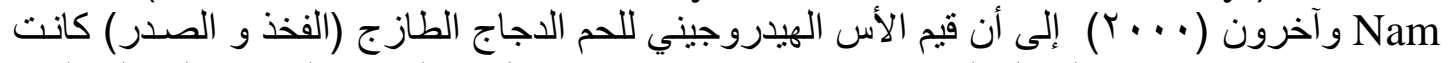

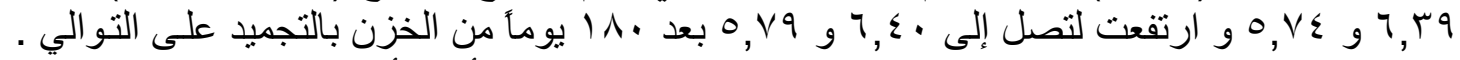

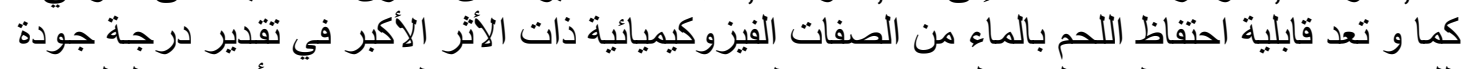

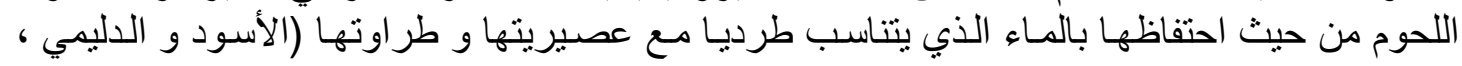

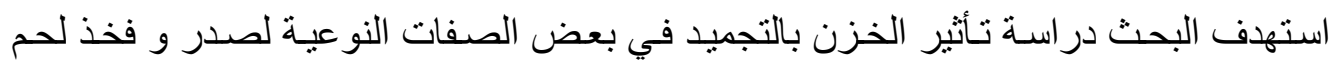

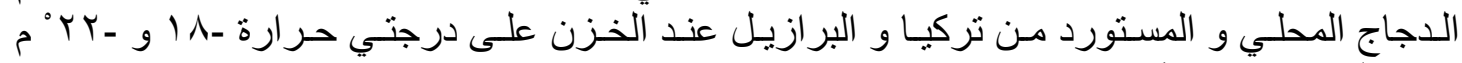

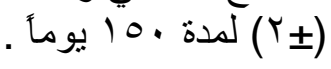

\section{مواد البحث و طرائقه}

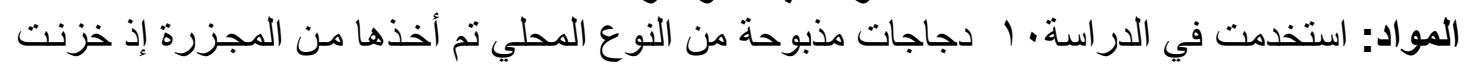

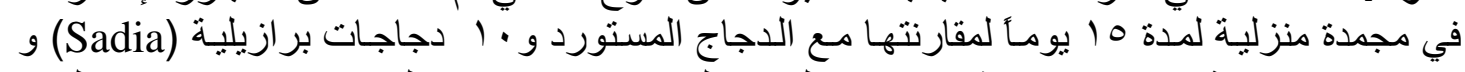

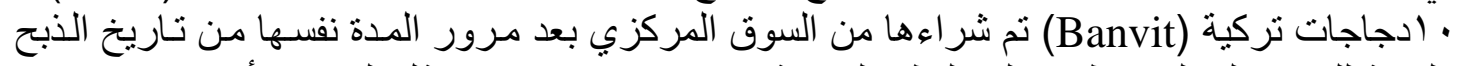

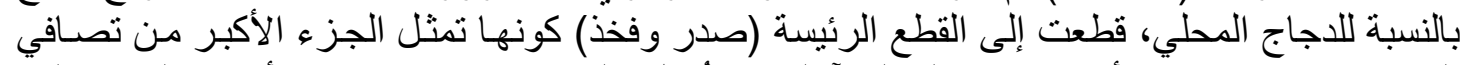

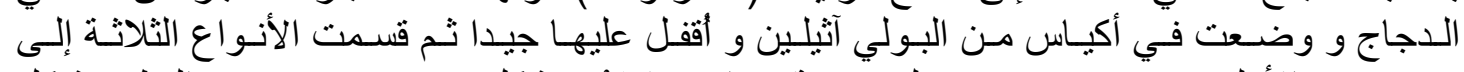

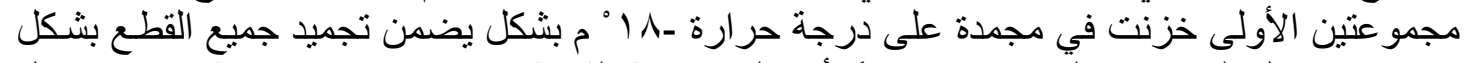

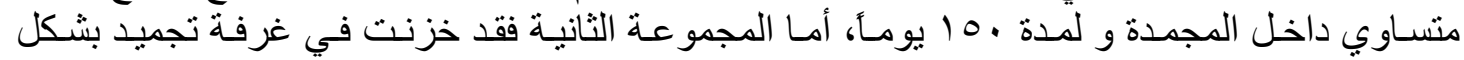
من دوران 


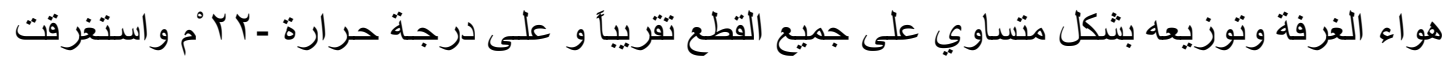

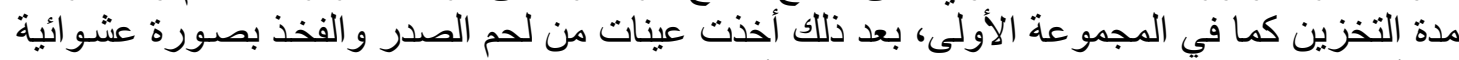

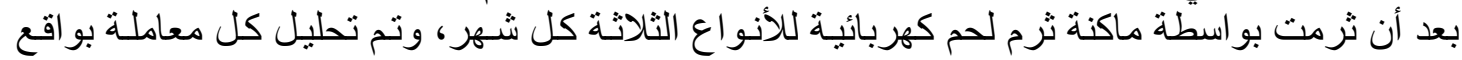
ثلاثة مكررات . ثلات

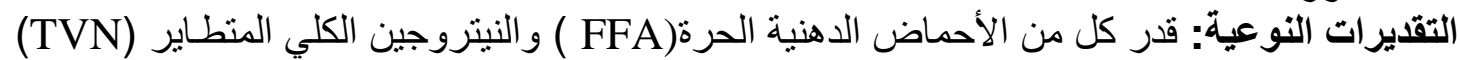

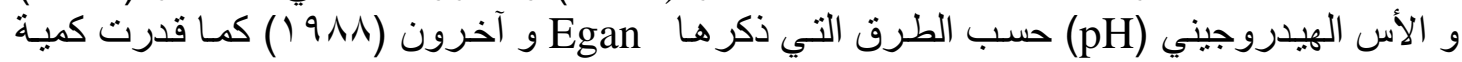
الماء المرتبط (WHC) حسب الطريقة التي ذكرها Anglmier و آخرون(

\% \%للماء المرتبط = \% للرطوبة الأصلية - \% \% للماء الحر في العينة .

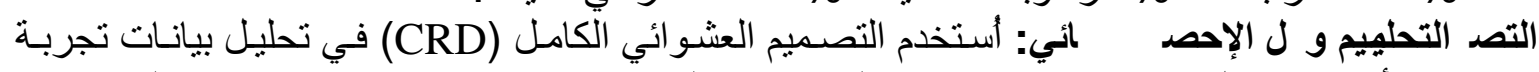

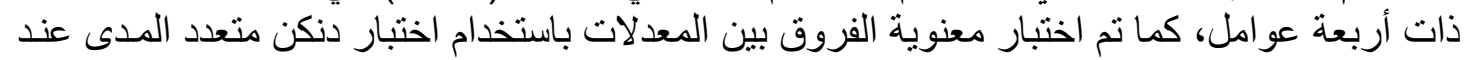

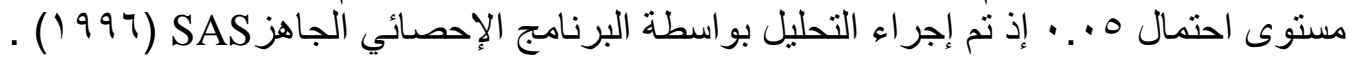

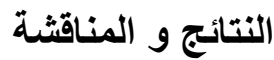

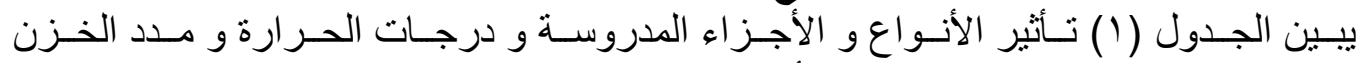

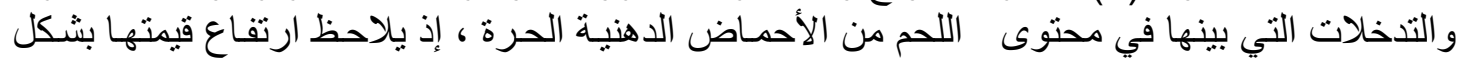

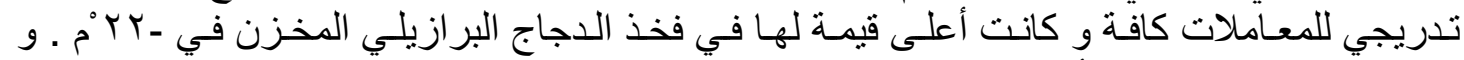

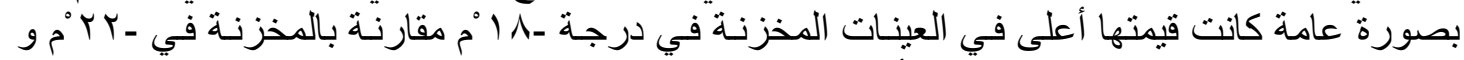

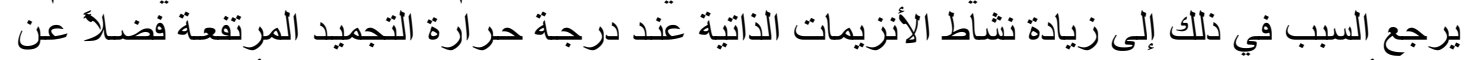

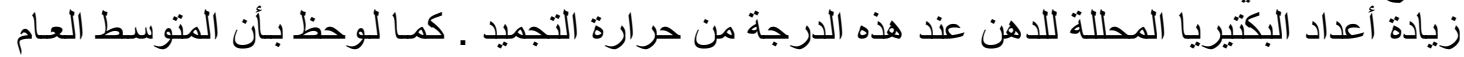

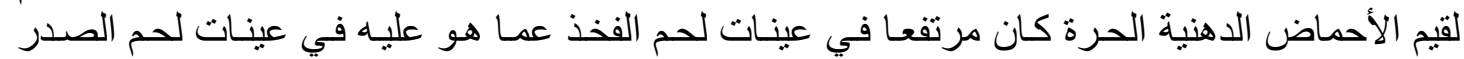

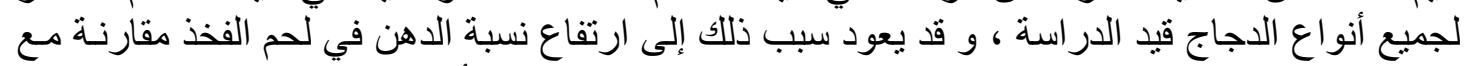

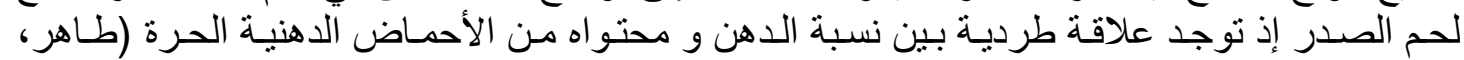

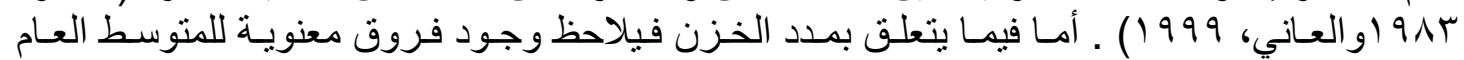

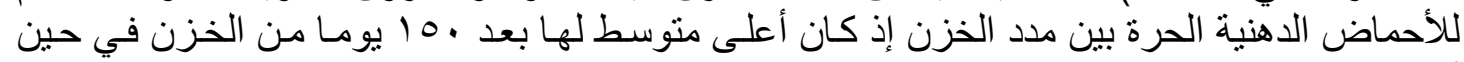

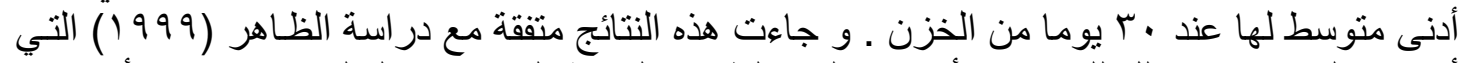

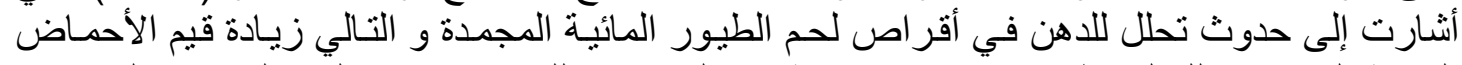

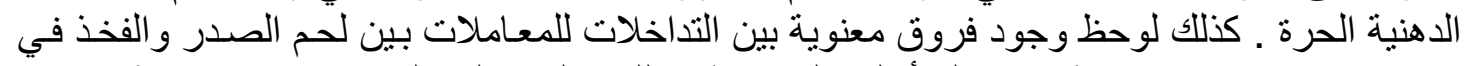

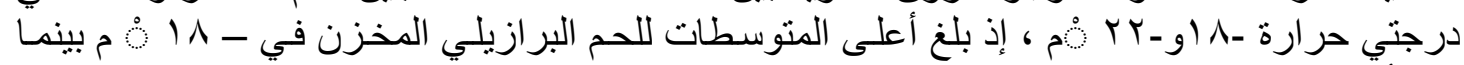

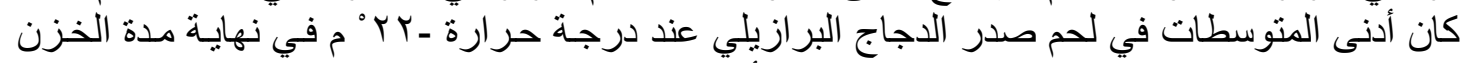

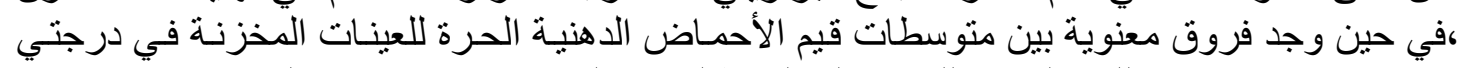

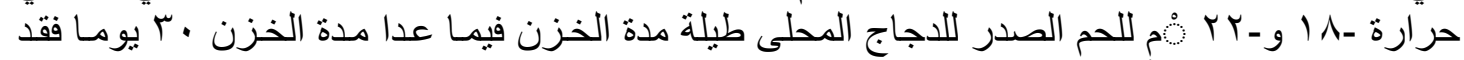

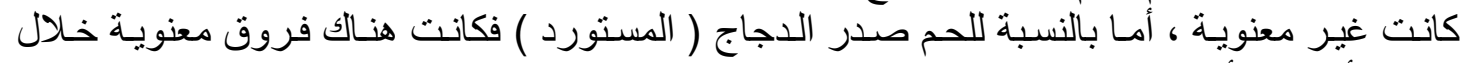

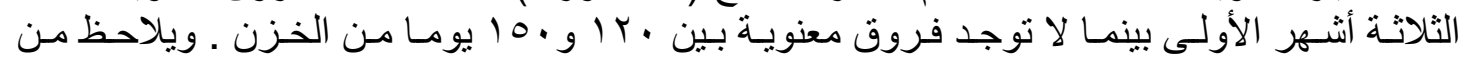

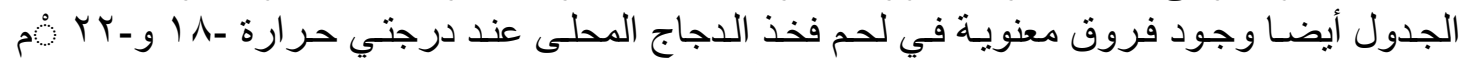

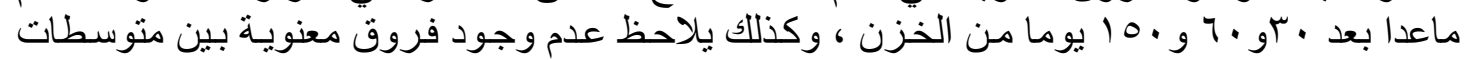

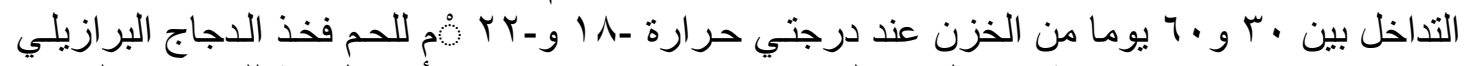

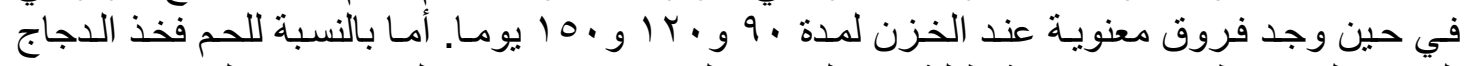

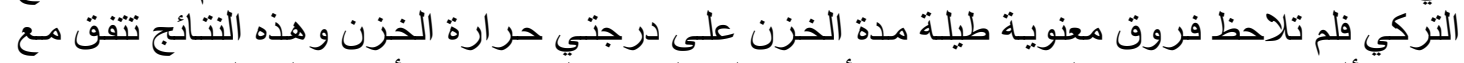

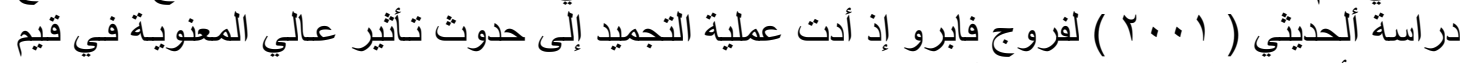

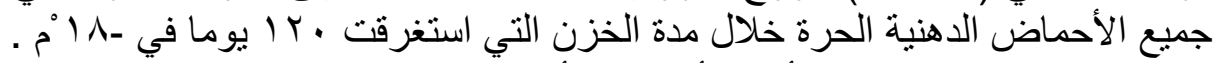

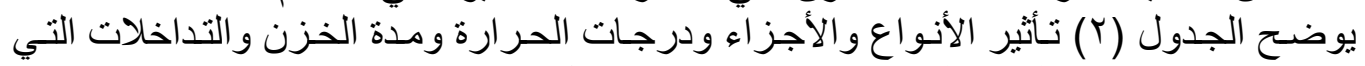

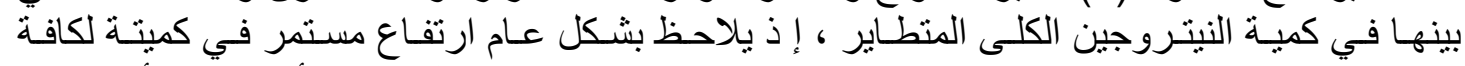

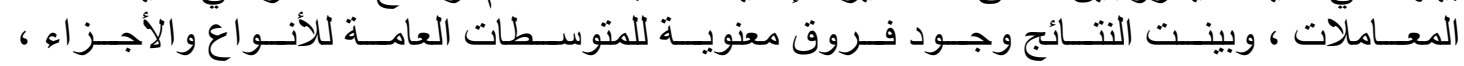



المجلد (דب) العدد (1) ^...
(ISSN 1815-316X)
مجلة زر اعة الر افدين

الجدول ( (1): المتوسطات 土 الخطأ القياسي لتأثير الأنواع والأجز اء المدروسة ودرجات حرارة التجميد و مدد الخزن والتداخلات التي بينها في النسبة المئوية للاحماض الدهنية الحرة

\begin{tabular}{|c|c|c|c|c|c|c|c|c|c|c|c|c|}
\hline \multirow{3}{*}{ الأنو اعن ×الأجز اء للتداء } & \multirow{3}{*}{ |الأنواع×الأجز اللتداخل xد } & \multirow{3}{*}{ 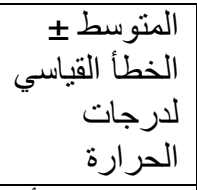 } & \multirow{3}{*}{ 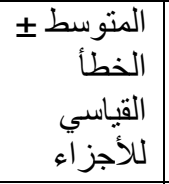 } & \multirow{3}{*}{ 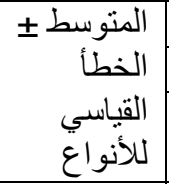 } & \multicolumn{5}{|c|}{ مدد الخزن بالأيام } & \multirow{3}{*}{ الحرارة } & \multirow{3}{*}{ الأجزاء } & \multirow{3}{*}{ الأنواع } \\
\hline & & & & & 10. & Ir. & 9. & 7. & \multirow[t]{2}{*}{$r \cdot$} & & & \\
\hline & & & & & & رن & و × درطات التداخل بات الحر & الأنواع × الأجز ال من & & & & \\
\hline \multirow[b]{2}{*}{ D. $q 4 q$} & b. 9V. & $\begin{array}{ll}1179 \\
. \quad .47\end{array}$ & \multirow[t]{2}{*}{$\begin{array}{ll} & .00 \\
\cdot & .09\end{array}$} & \multirow[t]{4}{*}{ 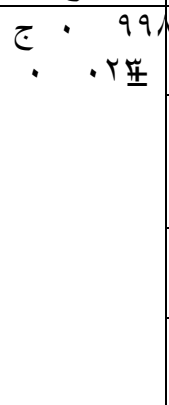 } & 1 & 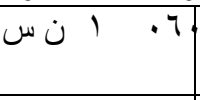 & י & ص 99 . رش & ثأ & م'11- & \multirow[b]{2}{*}{ الصدر } & \multirow{4}{*}{ دهوك } \\
\hline & . .9. & $\begin{array}{ll}11 \\
\end{array}$ & & & •. & \begin{tabular}{lll|} 
& 1 & $\cdots$ \\
& & \\
& &
\end{tabular} & , & 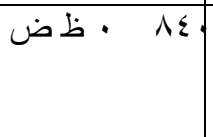 & I. v. & - & & \\
\hline & $g$ ' & & \multirow[t]{2}{*}{ 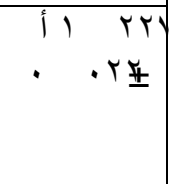 } & & . & • ب. & 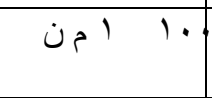 & ص & ل & ํ) 1 - & \multirow{2}{*}{ الفخذ } & \\
\hline $.0 \mathrm{~V}$ & $21 \cdot r$ & & & & ي & 10. & " & 90 & $ث \dot{\varepsilon} \cdot \quad \vee \wedge$ & 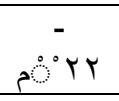 & & \\
\hline \multirow{2}{*}{ ا د } & j'. & & & $\begin{array}{ccc} & 1 & 17 \\
\cdot & .4 \underline{4}\end{array}$ & r'rq. & 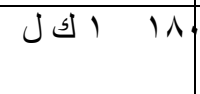 & י & ش & • & م'11- & \multirow{2}{*}{ الصدر } & \multirow[t]{4}{*}{ تركي } \\
\hline & $\tau^{\prime} \cdot r \mu$ & & & & ل & , & ص 99 & |ر & 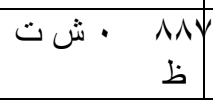 & $\begin{array}{l}- \\
\\
\end{array}$ & & \\
\hline \multirow[b]{2}{*}{. r r r } & r 1 ب & & & & ا & و 1 rq. & . & . 19 . & . 1. & $\dot{0}^{\circ} \Lambda_{-}$ & \multirow[b]{2}{*}{ الفخذ } & \\
\hline & ج I r & & & & ا & . & s & . 10. & . 9. & 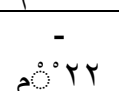 & & \\
\hline \multirow{2}{*}{ ا' } & $\begin{array}{lll} & Y\end{array}$ & & & \multirow[t]{4}{*}{$\begin{array}{ccc}1 & 1 & r t r \\
. & \cdot \underline{\underline{q}}\end{array}$} & أ0. & , & لر & 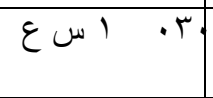 & ص ص & م'11- & \multirow{2}{*}{ الصدر } & \multirow{4}{*}{ برازيلي } \\
\hline & .1104 & & & & و & bح' & 10. & |' & . & $\begin{array}{l}- \\
\text { Sंrt }\end{array}$ & & \\
\hline \multirow[t]{4}{*}{ I I $\quad r \leq \varepsilon$} & I I r r. & & & & il Tr. & اب & . & لر & . & ํ) & \multirow{2}{*}{ الفخذ } & \\
\hline & 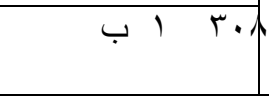 & & & & ب. & , إد & . & . 11 1. & •l 1. & - & & \\
\hline & \multicolumn{4}{|c|}{ متوسطات التداخل بين الأنواع × درجات الحرارة } & $\begin{array}{ll}1 & r y \\
. & . r \underline{z}\end{array}$ & $\begin{array}{ll} & \text { rog } \\
\cdot & \text { r }\end{array}$ & $\begin{array}{cc}T^{\prime} & |r| \\
\cdot & \cdot r \sharp\end{array}$ & $\begin{array}{ll} & \cdot r \phi \\
\cdot r \pm\end{array}$ & $\begin{array}{cc}0 \cdot & q r \\
\cdot & \cdot r q\end{array}$ & \multicolumn{3}{|c|}{ المتوسط 土 الخطأ القياسي } \\
\hline & & 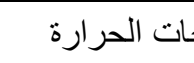 & & & & 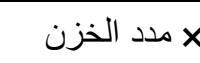 & 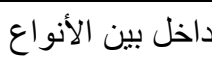 & متوسطات & & & 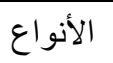 & \\
\hline
\end{tabular}




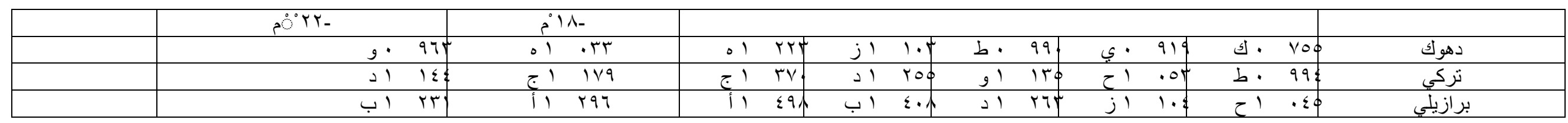

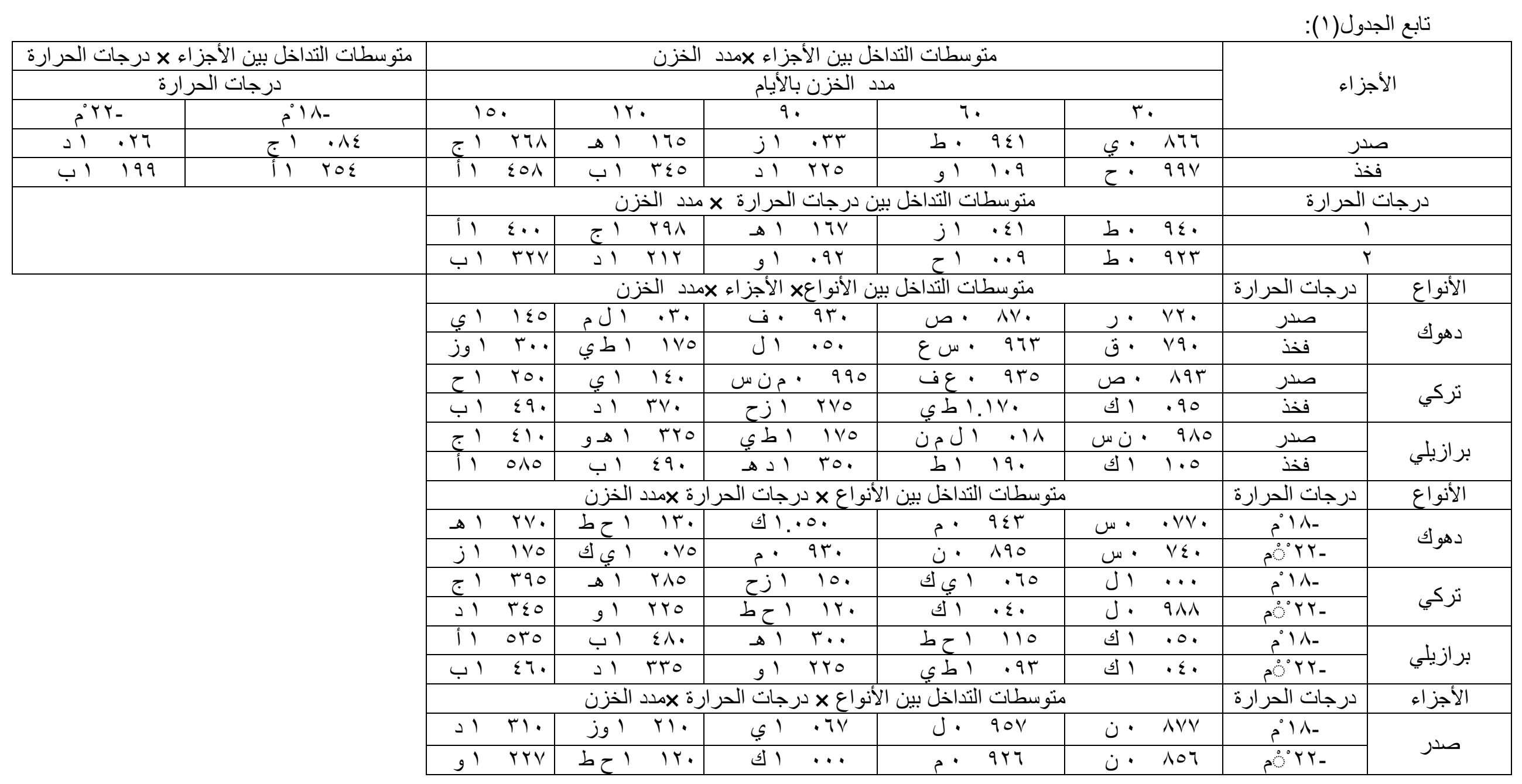




\begin{tabular}{|c|c|c|c|c|c|c|c|c|}
\hline$\{1 \leqslant 9$. & ا ج & TAV & ا هـ & $M M$ & $z^{\prime} \mid{ }_{1}$ & ك & مं1)- & ja \\
\hline $4 \leqslant r V$ & 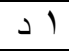 & $r \cdot r$ & jl & $1 \wedge r$ & "ا99. ا طي & 5. 99. & مंभr & حم \\
\hline
\end{tabular}




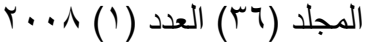
(ISSN 1815-316X)
مجلة زر اعة الرافين

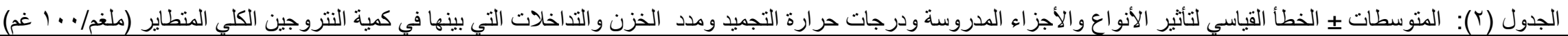

\begin{tabular}{|c|c|c|c|c|c|c|c|c|c|c|c|c|}
\hline \multirow{3}{*}{ اللألتداخل الأنواعز } & \multirow{3}{*}{ 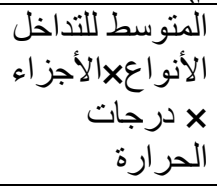 } & \multirow{3}{*}{ الدطأ القياسي } & \multirow{3}{*}{ 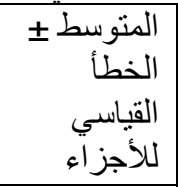 } & \multirow{3}{*}{ 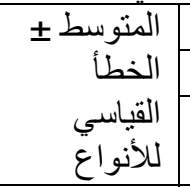 } & \multicolumn{5}{|c|}{ فتر ات الخزن بالأيام } & \multirow{3}{*}{ الحرارة } & \multirow{3}{*}{ 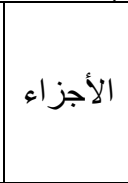 } & \multirow{3}{*}{ الأنواع } \\
\hline & & & & & 10 & Tr. & 9. & 7. & $r$. & & & \\
\hline & & & & & & x فترات الخزن & x درجات التداخل بين & الأنواع × الأج & & & & \\
\hline & $j l \varepsilon 9 \wedge 7$ & $\begin{array}{ll}110 & 1 \cdot 1 \\
0 & .4 \#\end{array}$ & $\mid 10.11$ & 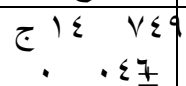 & $210 \quad 0.1$ & $510 \quad 11$ & $\varepsilon \omega \mid$ \& $q$. & | & J & 吕人- & & \\
\hline$\rightarrow 1=\lambda$ & b) & $\begin{array}{cc} & 9 V \\
. \quad 1 & .9 \\
\end{array}$ & · $\cdot r^{\prime}$ & & b & . & : & $ت$ ت & ل & - & & دهوك \\
\hline & $\tau^{\prime} \leqslant \quad \Lambda . r$ & & $4) \leqslant 997$ & & 10 أ. & . & . & ر) $1 \leqslant 9$. & |ت & ए। & & \\
\hline gle the & ك اي & & $\cdot . r \underline{q}$ & & . & , & ش & ظ $1 \leq r \Lambda$. & $\dot{\varepsilon}^{\prime} 1 \leqslant \quad r_{0}$. & קOr. & 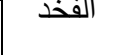 & \\
\hline & 10 197 & & & & . & j10 rA. & 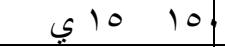 & J10.r. & . & ?11- & & \\
\hline (c) & $210 \quad 1 \leqslant 9$ & & & ماب اب & 1000. & j10 r. & 5 $10 \quad 1.1$ & م $1 \leqslant 99$. & 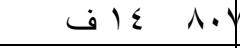 & مُrr- & الصفر & \\
\hline 1010 & $\rightarrow 10 \quad 1147$ & & & $\cdot . T \pm$ & 10 هـ. & b $10 \mathrm{rr}$ & 510.9. & $P^{1} \leq 9 \times 1$ & ع & iो人- & 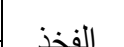 & لحتح \\
\hline J & $910.7 V$ & & & & 10 10 هـ & $b \tau^{10} \quad r$. & م) $1 \leqslant 997$ & ن $1 \leqslant 9 \leqslant$. & أ & ק्rr- & الهحت & \\
\hline 110 & i $10 \quad$ TVE & & & & $110 \quad 7 \leqslant$. & و 10 \&. & b $10 \quad 19$ & $510 \quad 1$. & J10. & PIN- & & \\
\hline 1,10 & ب $10 \quad r \leqslant \varepsilon$ & & & {$\left[\begin{array}{ll}110 & \text { YrY }\end{array}\right]$} & . & $910 \quad r 9$ & . & 5) 10.1 . & $\rho^{\prime} \varepsilon$ & קOrr- & الحص & \\
\hline 19 19 & & & & - $\cdot r^{4}$ & ج & $910 \mathrm{rA}$ & 10 10 إطي & 5) 10.1 . & م $1 \leqslant 99$. & $P^{\circ} \mid \Lambda_{-}$ & 1) & جر'يلي \\
\hline & $210 \quad 10 \%$ & & & & $210 \leqslant 1 t$ & j10 r. & $510 \quad 11$. & م) $1 \leqslant 99$. & $\varepsilon^{\prime} \leqslant \lambda \wedge t$ & PיYr- & القحد & \\
\hline & لم الحر ارة & أنواع × درجا: & 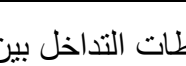 & & $110 \leqslant 0$ & $210 \quad 104$ & $P^{1} \leqslant 974$ & د) & $\rightarrow 1 \leqslant \quad V \leqslant$. & 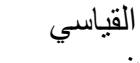 & 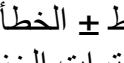 & 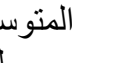 \\
\hline & & & & & $\cdot \cdot+\underline{\#}$ & 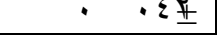 & $\cdot \cdot \varepsilon \underline{ \pm}$ & 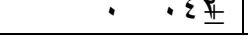 & - $\leqslant 0 \pm$ & & & \\
\hline & & ل ل الحرارة & & & & إتات الذن. & 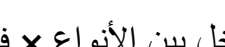 & مته سطات الات & & & إلانه |ع & \\
\hline & \% & & 1 & & & & & & & & & \\
\hline & و إ & $7 . r$ & أ & 190 & $210 . r \ldots$ & إي 10. & 5) 1 \& 704 & JI & $p^{\prime}\{r$ r & & دهوك & \\
\hline & 210 & 1.1 & (10 & 171 & ب 10 —91 & 010 ror & j10. & b) $\leqslant 910$ & ئ & & تركي & \\
\hline & 10 ب ب & 199 & 110 & TOS & 110074 & 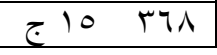 & $910 \quad 174$ & $2^{10} \cdot 7 \pi$ & b) \& $9 \times 17$ & & بر ازيلي & \\
\hline
\end{tabular}




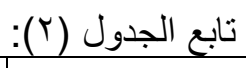

\begin{tabular}{|c|c|c|c|c|c|c|c|c|c|}
\hline بين الأجزاء × درجات الحرارة & 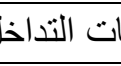 & منوسط & \multicolumn{5}{|c|}{ متوسطات التداخل بين الأجز اء × فترات الخزن } & \multirow{3}{*}{\multicolumn{2}{|c|}{ 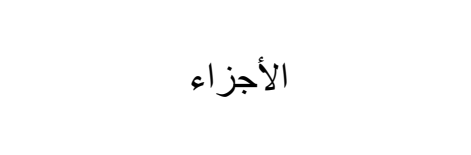 }} \\
\hline \multicolumn{3}{|c|}{ درجات الحرارة } & \multicolumn{5}{|c|}{ فتر ات الخزن بالأيام } & & \\
\hline 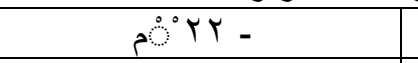 & 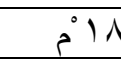 & & 10. & Ir. & 9. & 7. & $r \cdot$ & & \\
\hline |... & 110 & 104 & $110 \quad 011$ & ج $10 \quad r \cdot r$ & $\rightarrow 10 \quad \ldots v$ & jl & bl $\leq V V \varepsilon$ & \multicolumn{2}{|c|}{ صدر } \\
\hline \multirow[t]{4}{*}{$د \leq 94$} & 10 & $.0 \leqslant$ & ا & $210 \quad 11 r$ & $91 \leqslant 9 r 7$ & $\tau^{\prime} \leqslant \wedge r V$ & اي & \multicolumn{2}{|c|}{ فخذ } \\
\hline & & & \multicolumn{5}{|c|}{ منوسطات التداخل بين درجات الحرارة × فترات الخزن } & \multicolumn{2}{|c|}{ 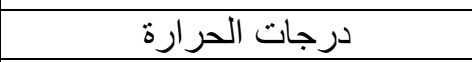 } \\
\hline & & & I10 Orr & إ. & ب r & و & $\tau^{\prime \leqslant 1.1}$ & \multicolumn{2}{|c|}{1} \\
\hline & & & ا & 210.94 & $j 1 \leqslant \quad \wedge 99$ & $\tau^{\prime} \backslash \wedge \lambda$ & b) & \multicolumn{2}{|c|}{ r } \\
\hline & & & \multicolumn{5}{|c|}{ متوسطات التداخل بين الأنو اع× الأجز اء × فترات الخزن } & 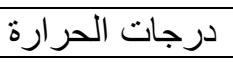 & 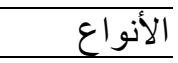 \\
\hline & & & 10 ro. & ع Is $9 r$ & . & 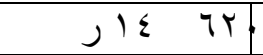 & ظ) $\leq \leqslant 0$. & صدر & \\
\hline & & & j10 ro. & ص 1 ا & ( & $ت 1 \leqslant \leqslant \wedge \phi$ & & فخذ & د دهوك \\
\hline & & & . & $910 \quad \mathrm{rq}$ & وا 10 10 & 1. & ف) 101 & صدر & \\
\hline & & & $210 \leqslant 10$ & $\tau^{10} \quad r^{\prime} \phi$ & J10. & عاس & & فخذ & تركي \\
\hline & & & $110 \quad 71$. & $210 \quad r 9 \phi$ & b $10 \quad 110$ & 510.9. & هوب. 10 من & صدر & \\
\hline & & & 10 014 & $10 \quad r \leqslant$ & 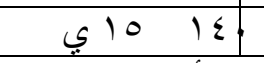 & |ه & $\varepsilon^{\prime} \leqslant 9 r y$ & فخذ & براريلي \\
\hline & & & & حر ارة × فتر ات الخ & ين الأنو اع × درجات & متوسطات التداخل & & درجات الحرارة & الأنواع \\
\hline & & & $210 \leqslant 0$. & 19. 10 من & 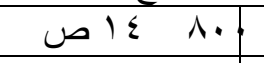 & & $j \leqslant 0.5$ & 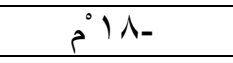 & \\
\hline & & & b $10 \quad 1 \leq 0$ & & $J \leq 0 . y$ & ش & $ت \quad r V \phi$ & POrt - & دهوك \\
\hline & & & . & jlo rop & إبا 10 10ي & 0. & ف) & 吕1/- & \\
\hline & & & ج $10 \leqslant 0$ & j10 ro. & J10. & (1) 97\$ & r & ק'rr- & لرحي \\
\hline & & & 110090 & $\rightarrow 10 \quad r q$. & $2^{10} 11$. & 510.9. & 10 10 10 من & ill- & \\
\hline & & & بr & $910 \quad r \leqslant \phi$ & b $10 \quad 1 \leqslant \phi$ & & $\varepsilon 1 \leqslant 94 Y$ & PיYr- & برازيلي \\
\hline & & & & حر ارة × فترات الذ & بن الأنواع × درجات & متوسطات التذاخل & & درجات الحرارة & الأجزاء \\
\hline & & & 110015 & 210 YTH & $j 10.1$ & b) $\varepsilon 9 \wedge r$ & $J 1 \leq 10$. & مं1 - & \\
\hline & & & ب $10 \leqslant r$ & $910 \quad 1 \leq$ & كا & م & س & יंr - & يدر \\
\hline & & & . & $10 \quad I V Y$ & bl $\leqslant 9 \wedge y$ & ك 1 \& $\wedge \wedge$ & ن 1 ( & 吕1- & فخذ \\
\hline & & & 10 TrA & $\tau^{10} \cdot \leqslant \psi$ & J1 & أ & $\varepsilon^{\prime} 1 \leqslant \quad 7 \leqslant$ & م्Yr- & وحد \\
\hline
\end{tabular}




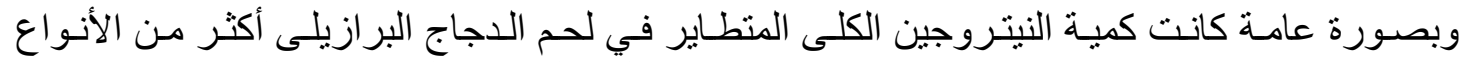

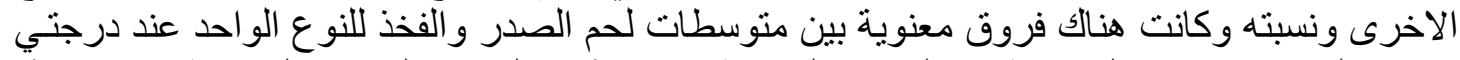

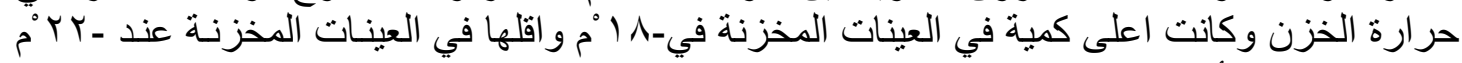

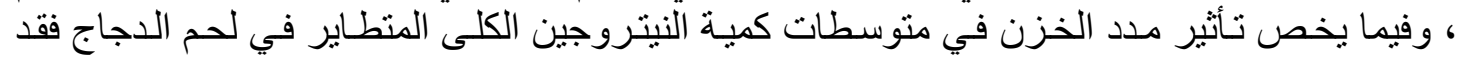

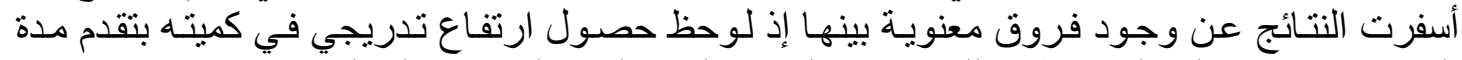

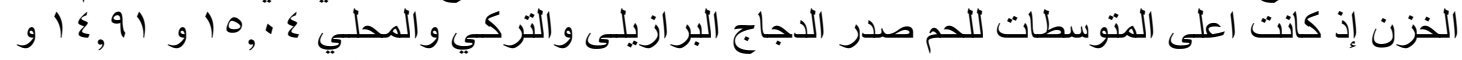

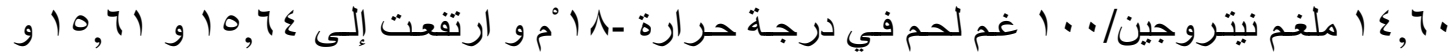

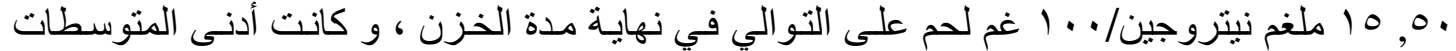

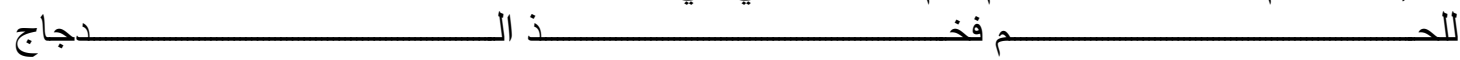

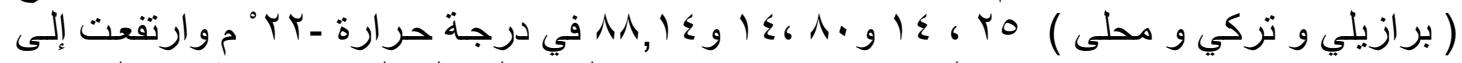

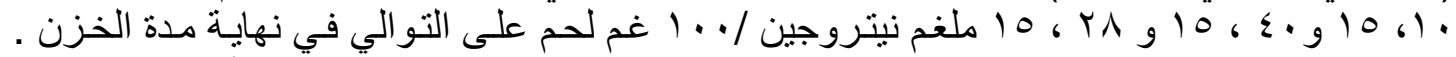

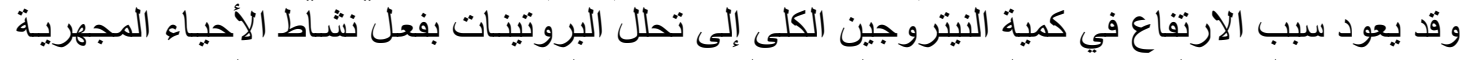

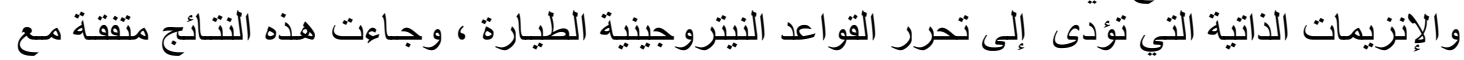

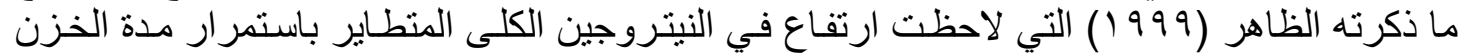

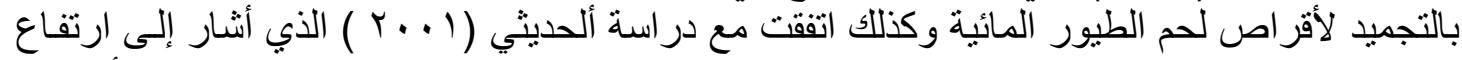

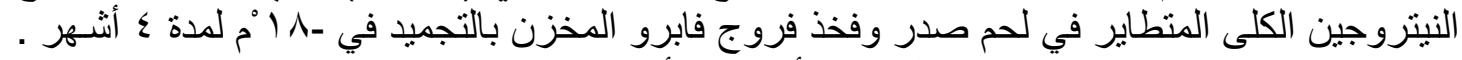

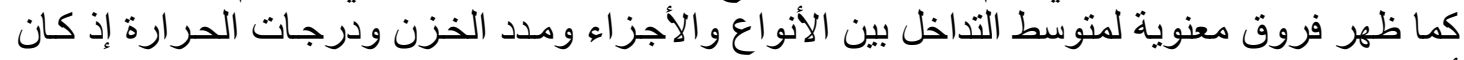

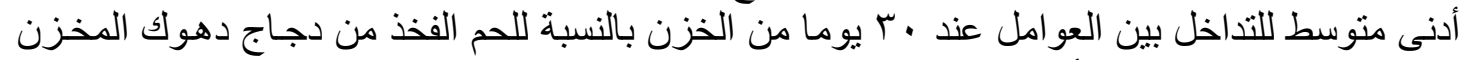

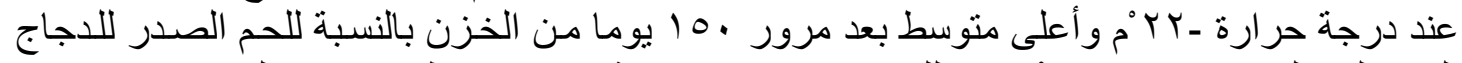

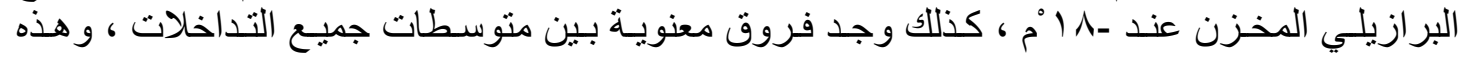

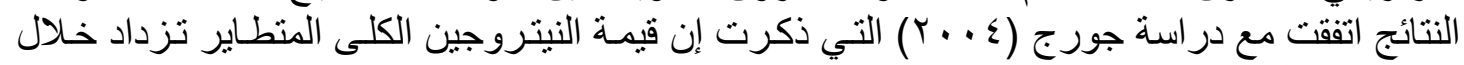

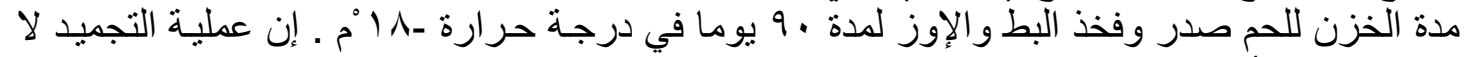

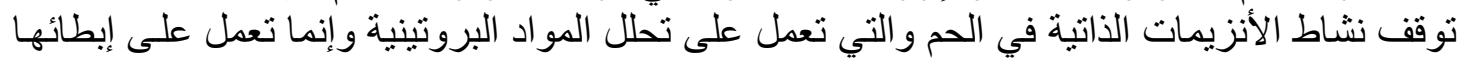

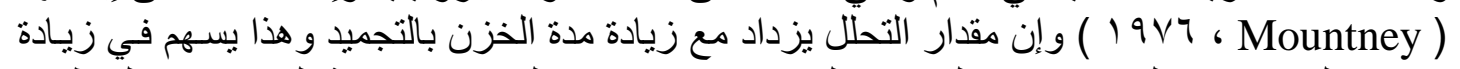

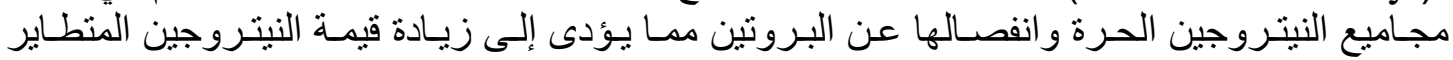

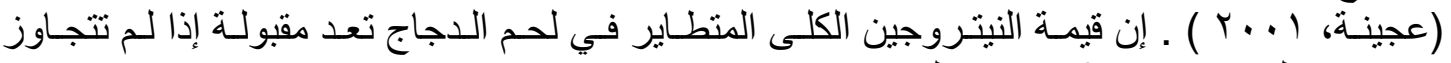

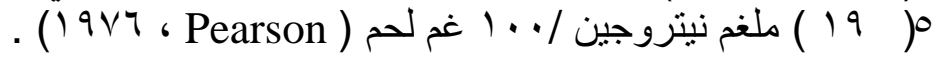

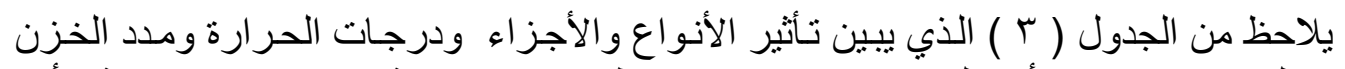

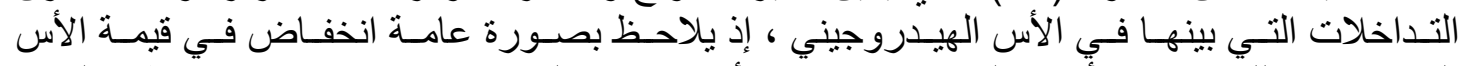

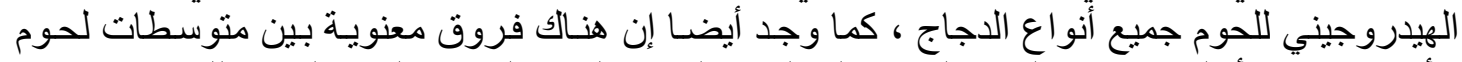

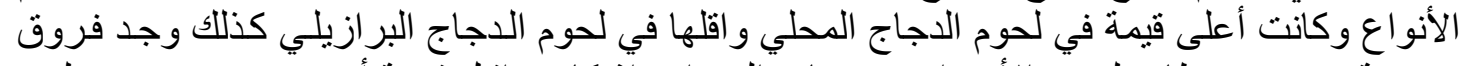

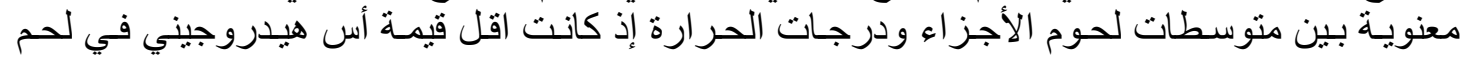

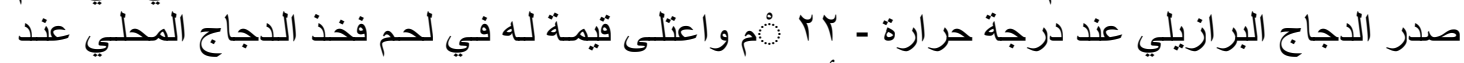

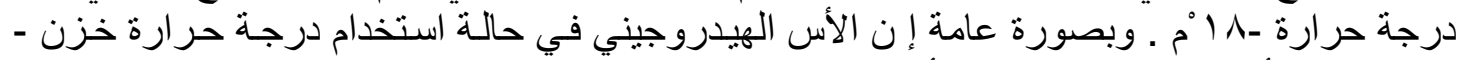

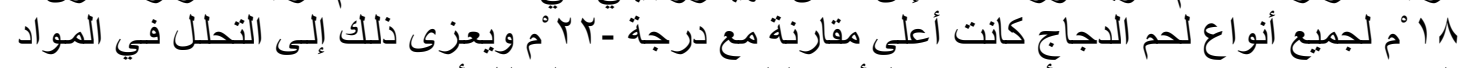

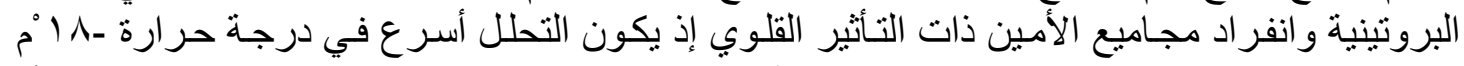

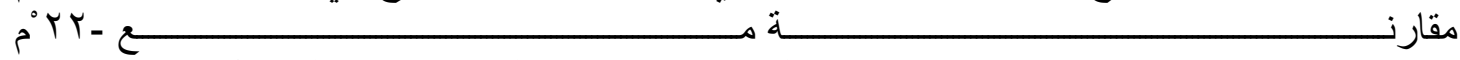

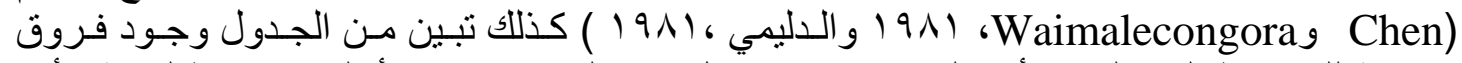

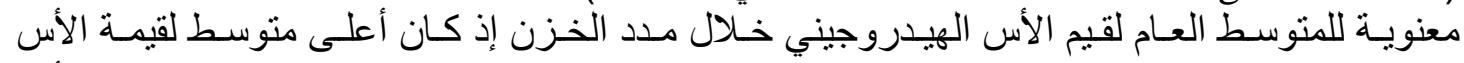

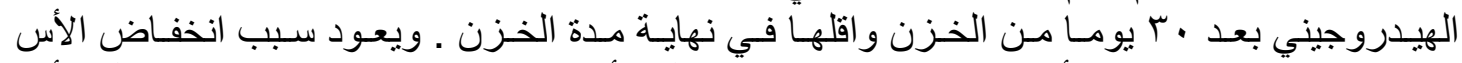

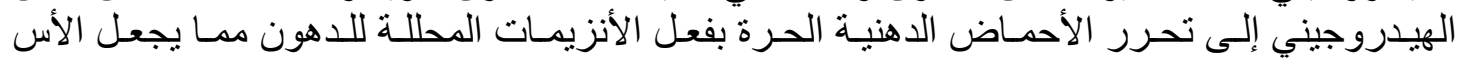

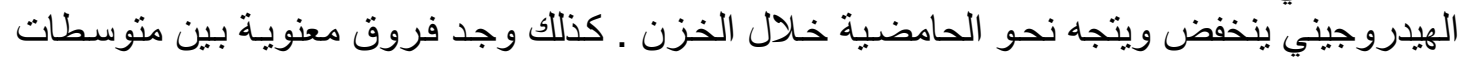

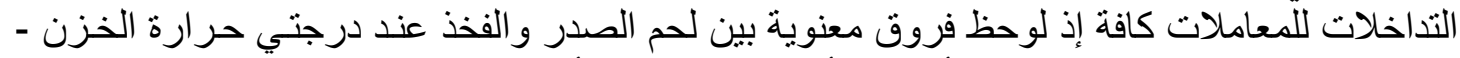

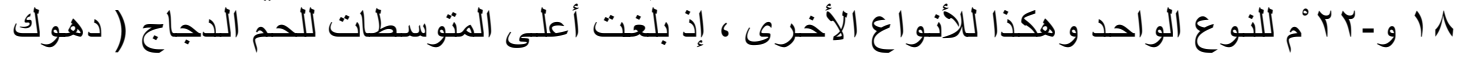




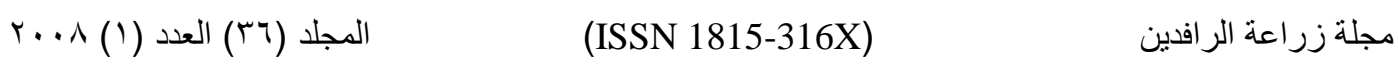

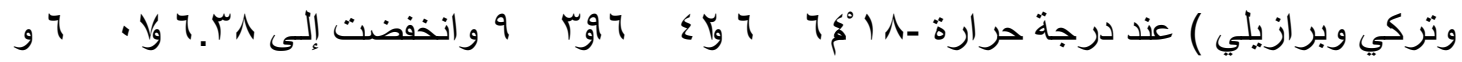

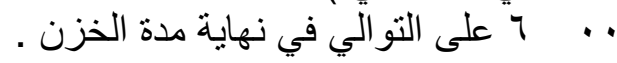


الجدول (r): المتوسطات 土 الخطأ القياسي لتأثير الأنو اعو الأجز اء المدروسة ودرجات حرارة التجميد ومدد الخزن و التداخلات التي بينها في الأس الهيدروجيني

\begin{tabular}{|c|c|c|c|c|c|c|c|c|c|c|c|c|}
\hline \multirow{3}{*}{ الأنو سط اللتدزاءل } & \multirow{3}{*}{ 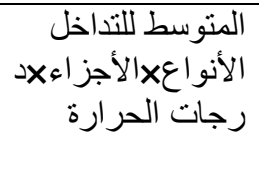 } & \multirow{3}{*}{ 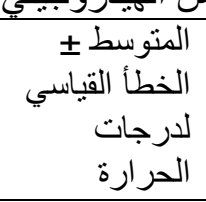 } & \multirow{3}{*}{ 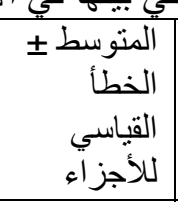 } & \multirow{3}{*}{ 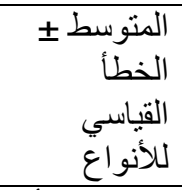 } & \multicolumn{5}{|c|}{ مدد الخزن بالأيام } & \multirow{3}{*}{ 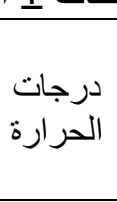 } & \multirow{3}{*}{ الأجزاء } & \multirow{3}{*}{ الأنواع } \\
\hline & & & & & 10 & $\pi$. & 9. & 7. & $r$. & & & \\
\hline & & & & & \multicolumn{5}{|c|}{ الأنو × الأجز اء × درجات التداخل بين × الحرارة مدد الخزن } & & & \\
\hline \multirow[t]{2}{*}{ rا } & ( & 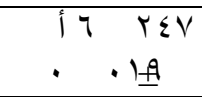 & \multirow[t]{2}{*}{$\varphi^{7} \cdot 94$} & \multirow[t]{4}{*}{ 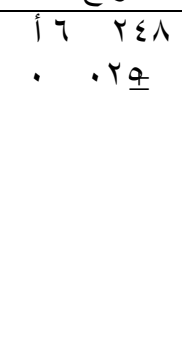 } & 19. & إ. & $j \tau r \ldots$ & g 97 r 7 . & د Y 1 \&r. & ए। & \multirow{2}{*}{ الصدر } & \multirow{4}{*}{ دهوك } \\
\hline & r & 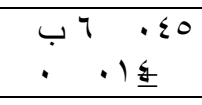 & & & ا & ظ 0 90. & .... & إ ص & . & PंYr- & & \\
\hline \multirow[t]{2}{*}{ IT Tr Tr } & II $\leqslant \wedge 7$ & & \multirow[t]{2}{*}{$\begin{array}{lc}17 & 199 \\
\cdot & .1 \pm \pm\end{array}$} & & $90 \quad 194$ & 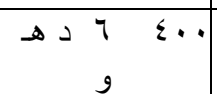 & ج 1 I & . & I 1 T $7 \varepsilon$. & مill- & \multirow{2}{*}{ الفخذ الفذ } & \\
\hline & A 1 I IVA & & & & J & ا & ل & & $\begin{array}{lll} & 1 & \Gamma . .\end{array}$ & PYr- & & \\
\hline \multirow[t]{2}{*}{4 I } & g 114. & & & ع إ & ض 9 ... & H 1 H & 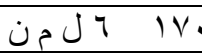 & r 1 ro. & j 1 r r r & 只1 & \multirow[b]{2}{*}{ الصدر } & \multirow{4}{*}{ تركي ل } \\
\hline & b 7 . & & & $\cdot \cdot r \pm$ & $\dot{\varepsilon} \dot{\varepsilon}^{\circ} \quad \wedge T$. & . & מ & إ ص & •l 10 1 نس & j & & \\
\hline \multirow[t]{2}{*}{$\begin{array}{ll}107 \\
\end{array}$} & ج & & & & r & |l ال من & $\tau^{1}$ ro. & ز & هـ & مان- & \multirow[t]{2}{*}{ الفخذ الفذ } & \\
\hline & $\tau^{7} \cdot 7 \varepsilon$ & & & & $\dot{H} \dot{\varepsilon}^{0} \quad 10$. & . & ، 1. 1 ص ق & . 17. & 27 ro. & PYr- & & \\
\hline \multirow[t]{2}{*}{ ه 7 I } & ju $1 . V$ & & & ' & $ث \dot{\varepsilon}^{\circ} \quad 10$. & ش 99. & & bح $\tau^{r} \quad r \leqslant$. & j & 只N- & \multirow[b]{2}{*}{ الصدر } & \multirow{4}{*}{ بر ازيلي } \\
\hline & 910 & & & $\cdot \cdot r \underline{Z}$ & ث0 & | 9 ه & ش & 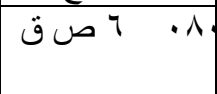 & إ & Prr- & & \\
\hline \multirow[t]{7}{*}{ (1) } & $\begin{array}{ll} \\
\end{array}$ & & & & ل... & ا & r. & j & . & مان- & \multirow{2}{*}{ الفذذ } & \\
\hline & . & & & & $\dot{H} 0 \quad \wedge r$. & . & ا & إن & ( & STr- & & \\
\hline & \multicolumn{4}{|c|}{ متوسطات التداخل بين الأنو اع × درجات الحرارة } & 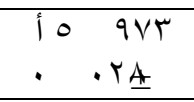 & $\begin{array}{l}27.09 \\
\cdot \quad \cdot r \underline{\underline{4}} \\
\end{array}$ & 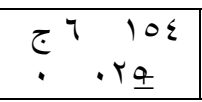 & 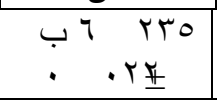 & 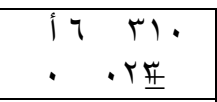 & \multicolumn{3}{|c|}{ الفتوسط 土 الخطأ القياسي } \\
\hline & \multicolumn{4}{|c|}{ درجات الحرارة } & \multirow{2}{*}{\multicolumn{5}{|c|}{ منوسطات التداخل بين الأنواع ×مدد الخزن }} & \multirow{2}{*}{\multicolumn{3}{|c|}{ الأنواع }} \\
\hline & PYr & & il & & & & & & & & & \\
\hline & T & $\cdots$ & if & $r 90$ & דו & g I IV. & $د \quad r \leqslant 0$ & "זוr & is r ro & & دهوك & \\
\hline & 7 & 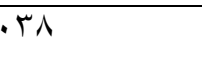 & ا & 11. & s) 0 9r. & bl ro & $\begin{array}{lll}j & \text { k }\end{array}$ & \begin{tabular}{lll|} 
& $Y$ & $Y$ \\
\end{tabular} & ( & & تركي & \\
\hline
\end{tabular}




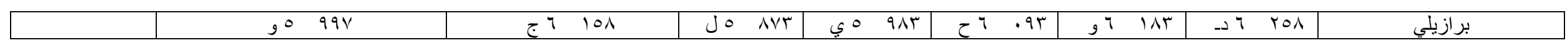




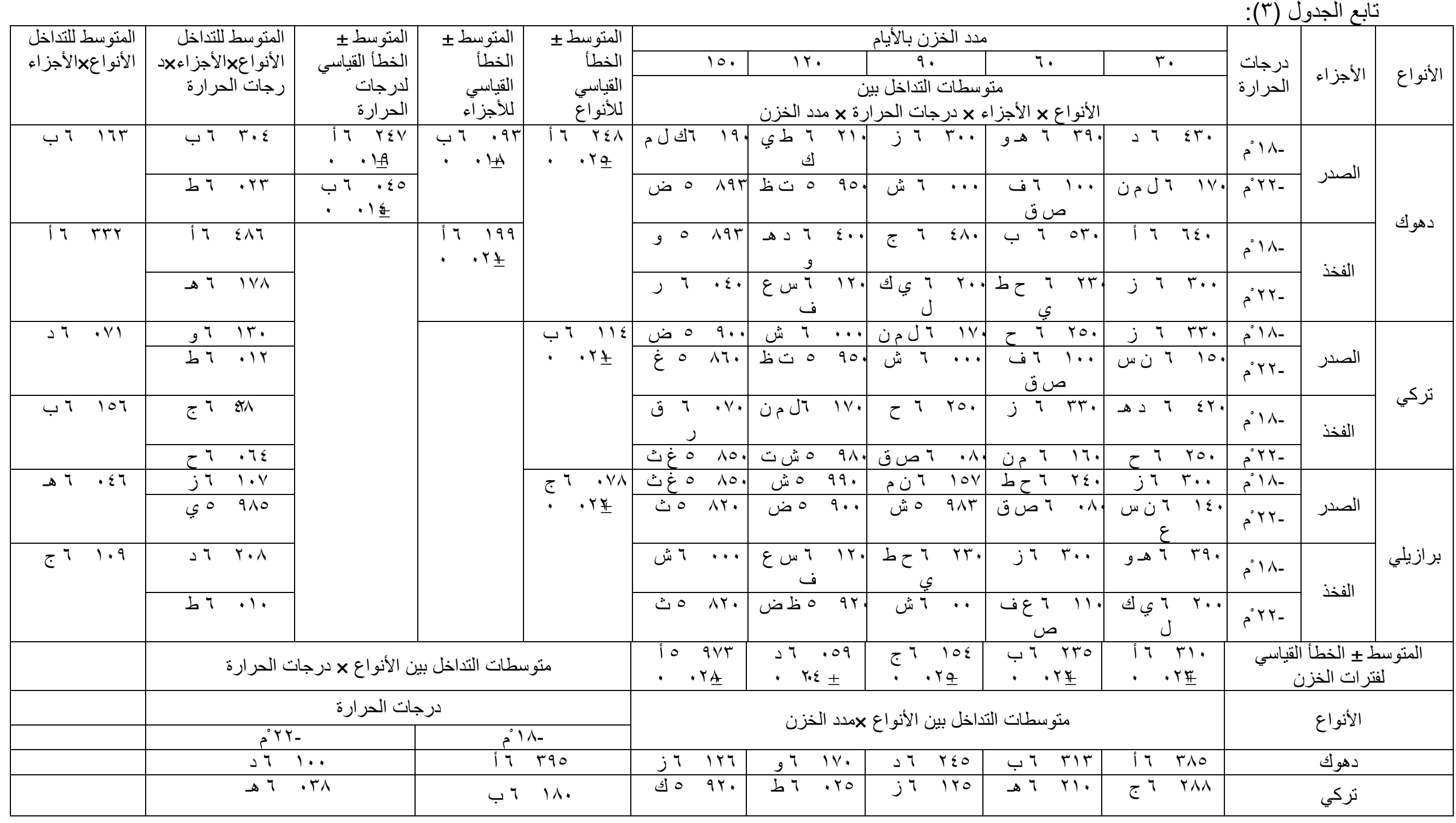




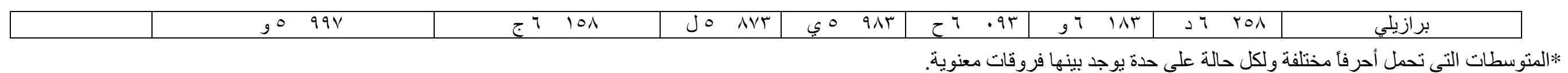




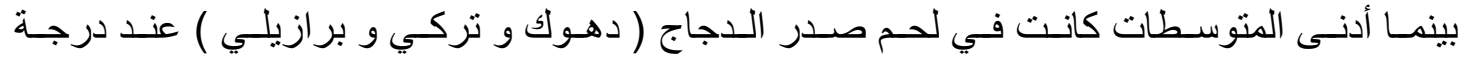

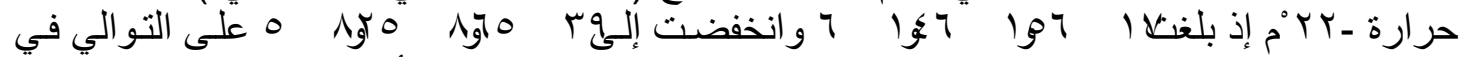

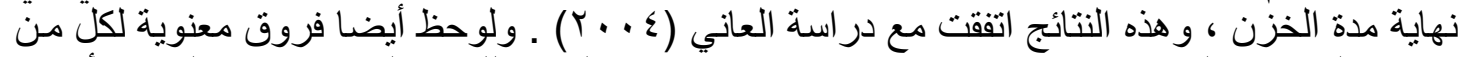

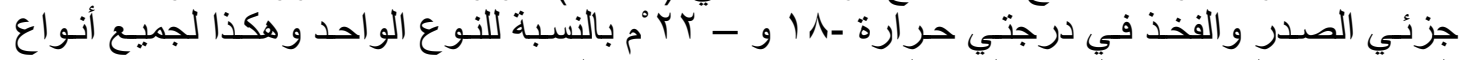

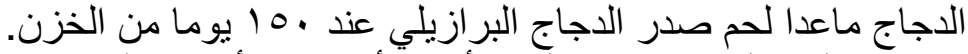

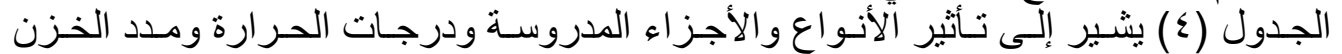

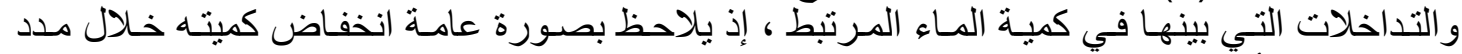

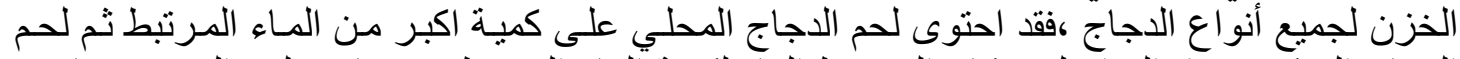

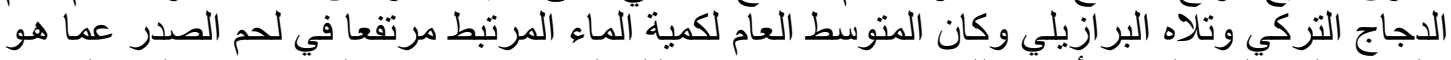

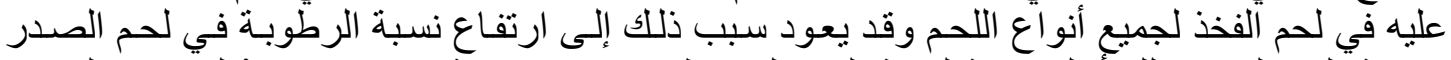

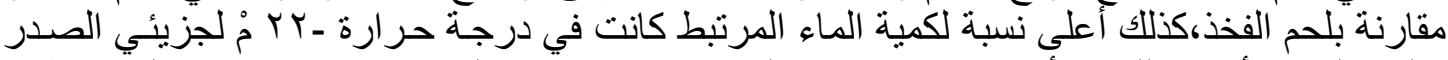

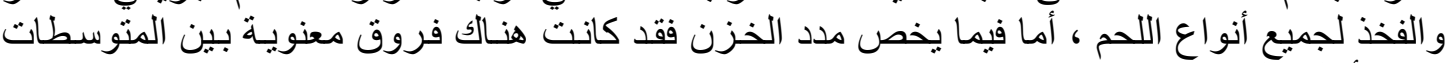

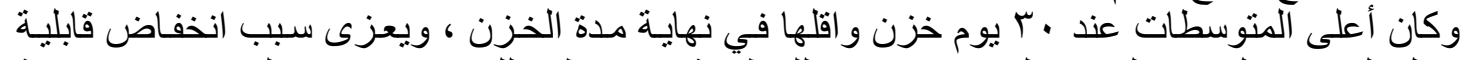

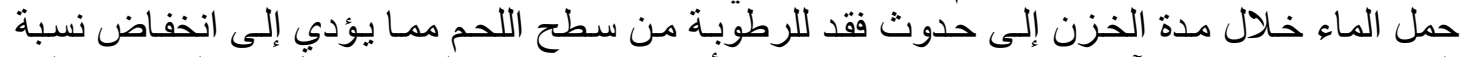

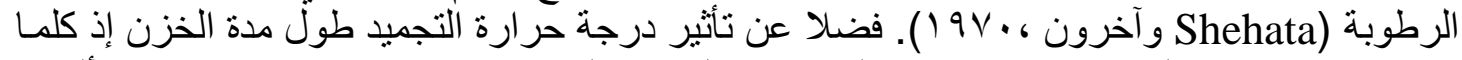

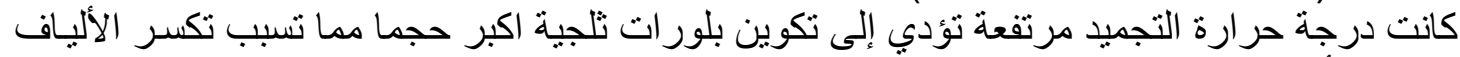

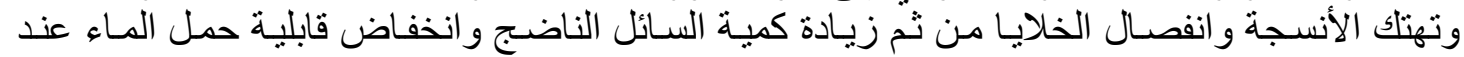

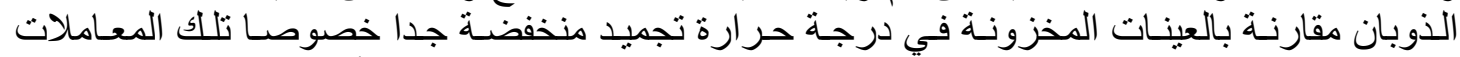

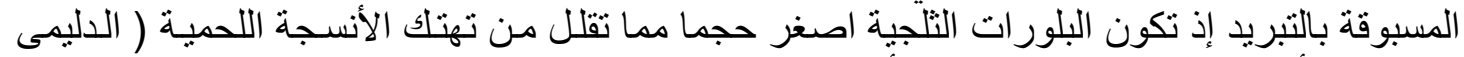

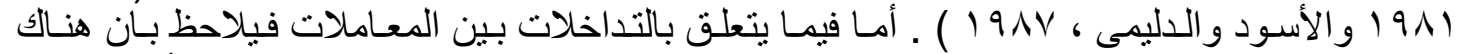

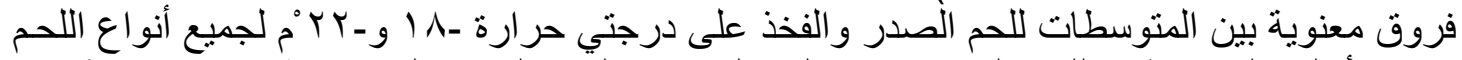

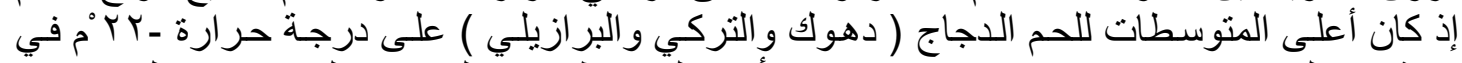

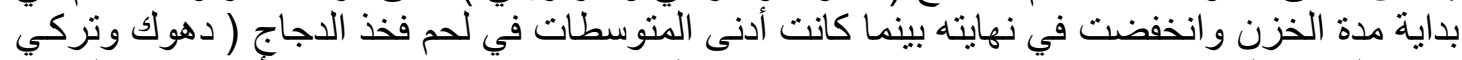

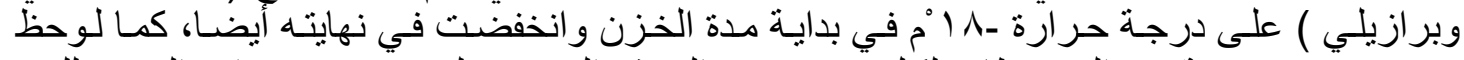

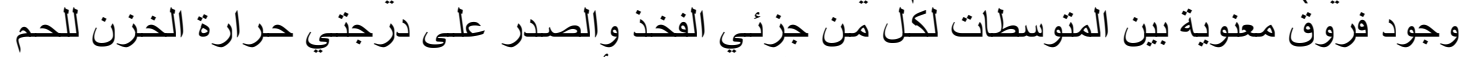

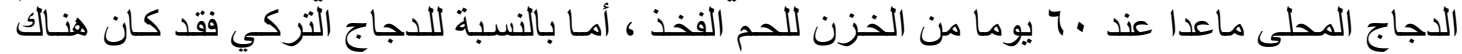

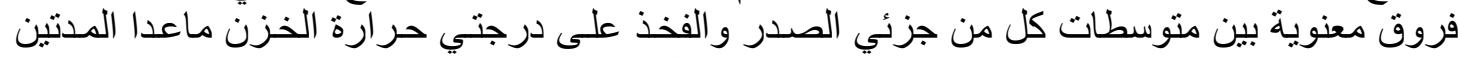

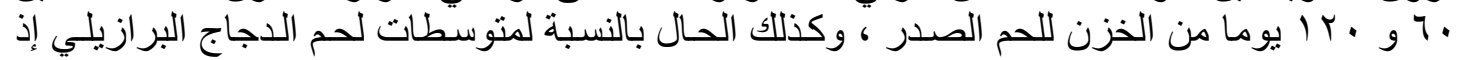

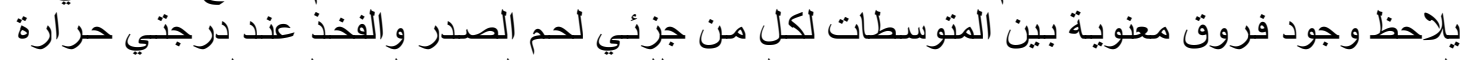

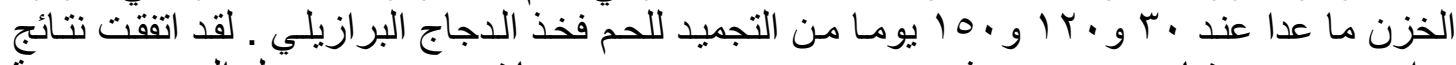

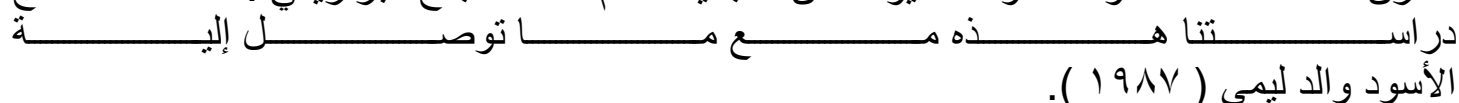

\title{
STUDY ON SOME ORGANOLEPTIC CHANGES OF LOCA L AND IMPORTED FROZEN CHICKEN MEATS
}

L.D.Al-Doori

F.S.Tamerkhan

Food Sci.Dept., College of Agric. And Forestry, Univ. of Mosul, Iraq

\begin{abstract}
This study is aiming to investigate the effect of freezing on some organoleptic Properties for kinds of Domestic, Imported (Turkish \& Brazilian) chicken meats at each thorax and thigh pieces which stored at -18 and $-22 \mathrm{c}$ for 150 days - Organoleptic properties analysis including total volatile nitrogen, free fatty acids, water holding capacity and $\mathrm{pH}$ were performed each 30 days. Results showed that Brazilian chicken meat contain higher total volatile nitrogen and free fatty acids comparing with other kinds of chicken meats where as $\mathrm{pH}$ value and water holding capacity were highest in Domestic comparing with Turkish and Brazilian chicken meats. While there are a significant $(\mathrm{P}<0.05)$ decrease in water holding capacity, $\mathrm{pH}$ and a significant $(\mathrm{P}<0.05)$ increase in free fatty acids
\end{abstract}




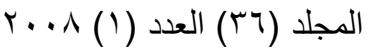
(ISSN 1815-316X)
مجلة زر اعة الر افدين

and total volatile nitrogen with increasing of freezing period for all kinds of chicken meats. 

المجلد (דب) العدد (1) ^...
(ISSN 1815-316X)
مجلة زر اعة الر افدين

الجدول ( ع ): المتوسطات 土 الخطأ القياسي لتأثنير الأنو اع والأجز اء المدروسة ودرجات حرارة التجميد و مدد الخزن والتداخلات التي بينها في كمية الماء المرتبط

\begin{tabular}{|c|c|c|c|c|c|c|c|c|c|c|c|c|}
\hline \multirow{3}{*}{ الأنو الاعطالأجزاء التجل } & \multirow{3}{*}{ 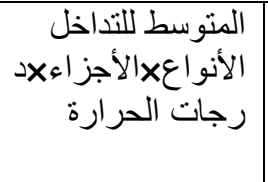 } & \multirow{3}{*}{ الدرجات الدرارة } & \multirow{3}{*}{ 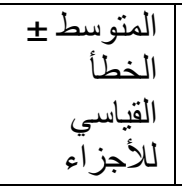 } & \multirow{3}{*}{ 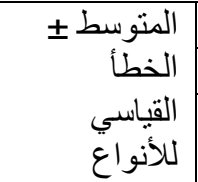 } & \multicolumn{5}{|c|}{ مدد الخزن بالأيام } & \multirow{3}{*}{ 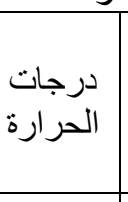 } & \multirow{3}{*}{ | الأجزاء } & \multirow{3}{*}{ الأنواع } \\
\hline & & & & & 10 & $T r$. & 9 & 7. & $r$. & & & \\
\hline & & & & & \multicolumn{5}{|c|}{ الأنو × الأجز اء × درجات التداخل بين الحرارة ×مدد الخزن } & & & \\
\hline \multirow[t]{2}{*}{$179.0 \leq$} & (1) & 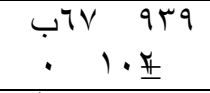 & \multirow[t]{2}{*}{\begin{tabular}{ll|l}
$\mid 71$ & $\leqslant 07$ \\
$\cdot$ & $\cdot \wedge \underline{\underline{4}}$
\end{tabular} \mid} & \multirow[t]{4}{*}{\begin{tabular}{cc|}
$\mid 71$ & $7 \pi 1$ \\
$\cdot$ & $\cdot 1$ 年
\end{tabular}} & STr & \begin{tabular}{rr|r}
$\omega$ & \\
$\varepsilon$ & & $0 .$. \\
\end{tabular} & j79 .0. & 27900. & ب9 & 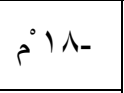 & \multirow{2}{*}{ الصدر } & \multirow{4}{*}{ د دهوك } \\
\hline & 179.97 & $\begin{array}{l}|71| \cdot 11 \\
\cdot 1 \cdot \underline{\pi} \\
\end{array}$ & & & ل11 ش & ك & $\begin{array}{lll}99 & 1\end{array}$ & ج 7979. & $179 \wedge 9$. & קיrr- & & \\
\hline \multirow[t]{2}{*}{ ج } & דT & & \multirow[t]{2}{*}{$\begin{array}{l}+9 V \quad \sum 9 \pi \\
\cdot \quad .9 \#\end{array}$} & & נד & |... & 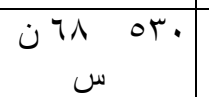 & Jir vo. & bru & مं। & \multirow[t]{2}{*}{ الفخذ ل } & \\
\hline & DTA TYT & & & & I IV Y... & |. & $\begin{array}{lll}71 & 7 . . \\
\end{array}$ & ل & 27190. & OY. & & \\
\hline \multirow[t]{2}{*}{ סrr r } & $د 7 \Lambda \quad M \varepsilon$ & & & \multirow[t]{4}{*}{$\begin{array}{c}\cdot \quad \cdot r \pm \\
\cdot T V R r\end{array}$} & 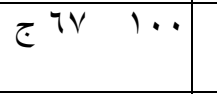 & |... & 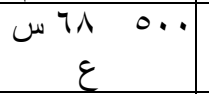 & b & •V. & 巳门人- & \multirow{2}{*}{ الصدر } & \multirow{4}{*}{ تركي } \\
\hline & ج r rov & & & & ب & מ & | & $\begin{array}{lll}\text { b } & 91 & 91 .\end{array}$ & $\rightarrow 79 \quad \%$ & קTr- & & \\
\hline \multirow[t]{2}{*}{ ه } & b TV .97 & & & & $\begin{array}{llll}\text { b } & \ldots\end{array}$ & |7r & $\begin{array}{lll}\mathrm{T} & 1\end{array}$ & $\begin{array}{lll}\dot{\varepsilon}^{T V} & 0 .\end{array}$ & (1. & pill- & \multirow[t]{2}{*}{ الفخذ } & \\
\hline & $z^{7 V} 119$ & & & & $\tau^{71} \quad .0$. & $\rightarrow 74 \quad \wedge \varepsilon \cdot$ & ITV $\quad$ r... & ל TV 70. & . & PYY- & & \\
\hline \multirow[t]{2}{*}{ ב ב } & jTV $9 \Psi V$ & & & \multirow{4}{*}{$\begin{array}{cc}e^{T V} 019 \\
\cdot 1 \cdot \#\end{array}$} & $د 7 V \quad \cdots$ & ث TV $\leqslant 0$. & מר ش ش.... & $\varepsilon^{7 \Lambda} \leqslant T V$ & ل & $\dot{P}^{\circ} \Lambda_{-}$ & \multirow{2}{*}{ الصدر } & \multirow{4}{*}{ بر ازيلي } \\
\hline & gTh $\cdot$. Tr & & & & ج & |. T T ע ע & 10. & ن & . & PYr- & & \\
\hline \multirow[t]{8}{*}{ gTV . . } & דr. VT ي & & & & b 70 91. & j74 $\quad$ V.. & ج & 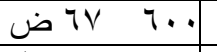 & ت TV & Pं। & \multirow{2}{*}{ الفخذ الفذ } & \\
\hline & & & & & b74 & j7r VT. & $\begin{array}{lll}I & T V & \text { Tr. }\end{array}$ & b TV 70. & ت T & PYr- & & \\
\hline & \multicolumn{4}{|c|}{ متوسطات التذاخل بين الأنواع × درجات الحرارة } & $\begin{array}{lll}49799 \\
\cdot \quad 14 \#\end{array}$ & 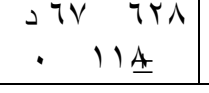 & 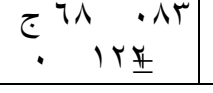 & 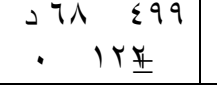 & $\begin{array}{l}171 \\
. \quad 111 \pm \\
\end{array}$ & \multicolumn{3}{|c|}{ المتوسط د الخطأ القياسي } \\
\hline & & نات الحرارة & & & & xدد الخزن & داخل بين الأنواع & منوسطات ال & & & الأنواع & \\
\hline & Prr & & $e^{01}$ & & & & & & & & & \\
\hline & 171 & VII & & $\pi Y$ & |.1. & $\rightarrow 7 \Lambda \quad r o$. & ج & بr & I79 rVo & & دهوك & \\
\hline & P TV & VV. & دTV & $V .0$ & م 77 OV7 & STV 810 & bTV $A \Gamma \wedge$ & $j 71 \quad r \varepsilon \cdot$ & . & & تركي & \\
\hline & ه TV & 001 & g TV & $\varepsilon \wedge V$ & ن. & JA & |V & $\tau^{71} \cdot .7 \varepsilon$ & g qu $\quad r .$. & & بر ازيلِي & \\
\hline
\end{tabular}


تابع الجدول (๕):

\begin{tabular}{|c|c|c|c|c|c|c|c|c|c|}
\hline 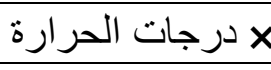 & 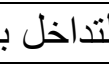 & متوسطات & & xدد الخزن & التداخل بين الأجز & متوسطات & & \multirow{3}{*}{\multicolumn{2}{|c|}{ الأجز اء اء }} \\
\hline \multicolumn{3}{|c|}{ 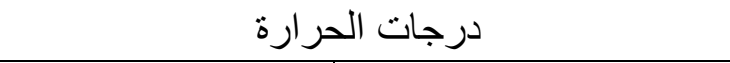 } & \multicolumn{5}{|c|}{ 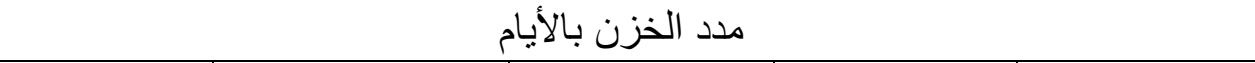 } & & \\
\hline Pיtr- & \multicolumn{2}{|c|}{ 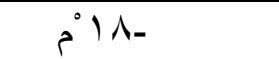 } & 10. & Ir. & 9. & 7. & $r \cdot$ & & \\
\hline I $71 \leqslant 94$ & 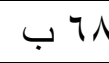 & $\sum Y 1$ & $\tau^{T V} \leqslant Y T$ & هـ $71.7 Y$ & ج 71 os & ب9 79 & $179 \quad r \leqslant r$ & \multicolumn{2}{|l|}{ صدر } \\
\hline \multirow{23}{*}{ ج TV Or. } & $د 7 V$ & $\{07$ & ي r TrY & b 7V 19r & jTV TYO & $97 \times \quad 911$ & $د \neg \Lambda \quad Y \wedge V$ & 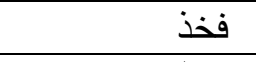 & \\
\hline & & & \multicolumn{5}{|c|}{ متوسطات التداخل بين درجات الحر ارة xدد الخزن } & \multicolumn{2}{|c|}{ درجات الحرارة } \\
\hline & & & ي 1111 & C $7 V$ ovo & $9 \uparrow \cdot \leq V$ & $271 \leq 71$ & ب & \multicolumn{2}{|l|}{1} \\
\hline & & & b 77911 & jTV Tג. & ه 71 Ir. & ج Th orv & $171 \quad 1 \ldots$ & \multicolumn{2}{|l|}{ r } \\
\hline & & & \multicolumn{5}{|c|}{ متوسطات التداخل بين الأنواع× الأجز اء xمدد الخزن } & درجات الحرارة & الأنواع \\
\hline & & & J71 $1 \cdot r$ & $\tau^{71} 70$. & $279 \cdot 10$ & ب9. & $179 \quad 1 Y 0$ & صدر & \multirow[b]{2}{*}{ د دهو } \\
\hline & & & ش 1. 1 IV & 571.0. & b71 070 & j7^ V70 & $\rightarrow 71970$ & فخذ & \\
\hline & & & JV ITV & $\dot{4} 71 \cdot 1 \cdot$ & ي & $\rightarrow 71 \quad 9.0$ & ج $7911 \cdot$ & صدر & \multirow{2}{*}{ 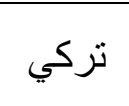 } \\
\hline & & & $\dot{\varepsilon} 77 \cdot$ ro & ظ 77 Nr. & ر 10. & ف TV OVo & 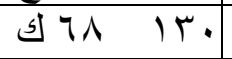 & فخذ & \\
\hline & & & $ت 7 V \quad .0$. & ص TV OYo & 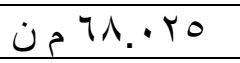 & | & $971 \times 90$ & صدر & \multirow{2}{*}{ بر ازيلي } \\
\hline & & & $\$ 70 \quad 99$. & . & G 7 V 17. & $\varepsilon 7 V \quad 7 r 0$ & س 7.0 & فخذ & \\
\hline & & & \multicolumn{5}{|c|}{ متوسطات التداخل بين الأنواع × درجات الحرارة ×مدد الخزن } & درجات الحرارة & الأنواع \\
\hline & & & ت 7 V & Jis ro. & $971 \quad \vee 9$. & 27910. & •r r 79 ب & $i^{\circ} 11$ & \multirow{2}{*}{ دهوك } \\
\hline & & & |. & b $71 \leqslant 0$ & $\rightarrow 71 \quad 10$. & ج r9 ro & I $79 \leqslant Y \cdot$ & S Yr- & \\
\hline & & & $\begin{array}{ll}* 77 & 00\end{array}$ & $ت 7 V \quad \varepsilon$. & فTV & $\begin{array}{lll}7 \lambda & Y . .\end{array}$ & $\tau^{71}$ ovo & 定1 & \multirow{2}{*}{ تركي } \\
\hline & & & $\dot{\varepsilon} 77 \quad 7.1$ & ش IV & $\varepsilon^{T V} \wedge \vee 0$ & $57 \wedge \quad r \wedge$. & j71 770 & P'rr- & \\
\hline & & & $177 \leqslant 9$. & 0 . . TV & JV 00. & Tr & S $71 \quad r \wedge 0$ & م'11- & \multirow{2}{*}{ بر ازيلي } \\
\hline & & & $ث 77 \quad 00$ & ל & סדו ד מע מע & ن 71.90 & |ه إل & S YYK- & \\
\hline & & & \multicolumn{5}{|c|}{ متوسطات التداخل بين الأنواع × درجات الحرارة × مدد الخزن } & 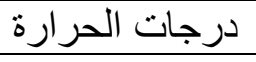 & الأجزاء \\
\hline & & & W TV & S TV $9 \wedge r$ & $97101 \mathrm{~V}$ & $د 7 \wedge \quad 9 V Y$ & ب r... & $\dot{5}^{\circ} \Lambda_{-}$ & \multirow[b]{2}{*}{ صدر } \\
\hline & & & س 〔V ミ1 & b 71 ls. & $\rightarrow 71$ OTV & ج $79 \cdot \leq V$ & I 79 rAV & S.rt- & \\
\hline & & & ق & ف7V ITV & $\dot{T}$ TV OVV & J 7890. & $\tau_{71} \quad r \uparrow$. & $\dot{P}^{\circ} \wedge-$ & \multirow{2}{*}{ 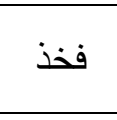 } \\
\hline & & & $\varepsilon^{T V} r r^{\prime}$ & 5 TV TVt & 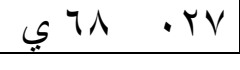 & j71 & & PRr- & \\
\hline
\end{tabular}




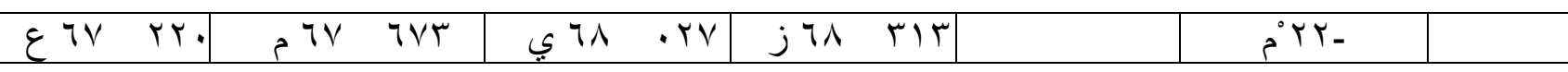




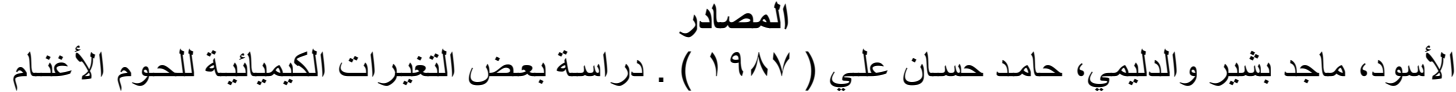

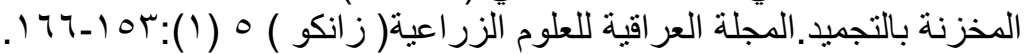

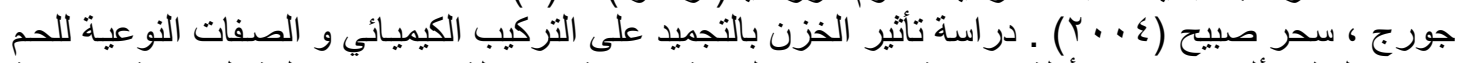

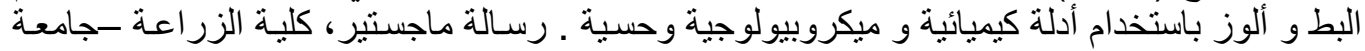

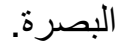

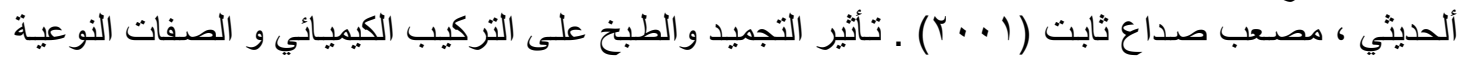

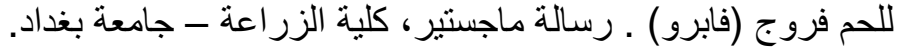

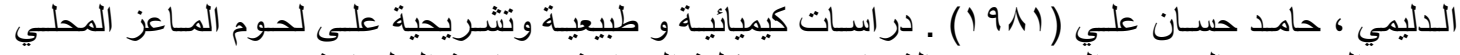

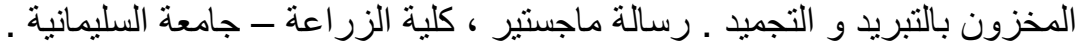

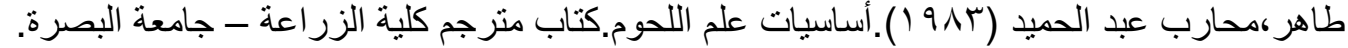

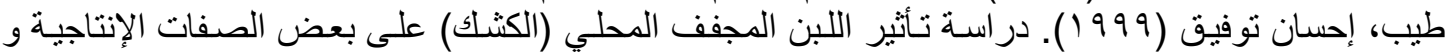

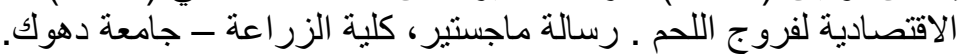

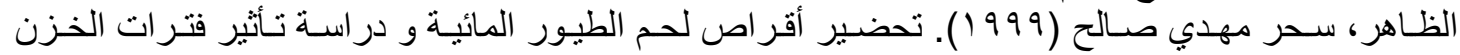

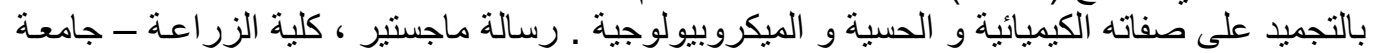

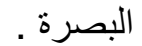

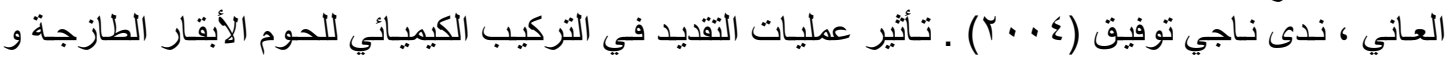

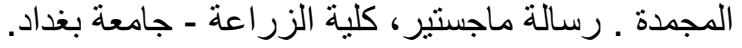

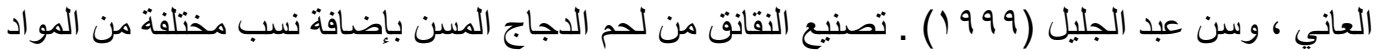

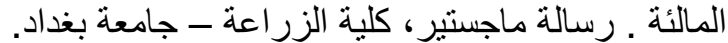

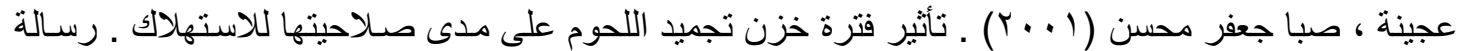

$$
\begin{aligned}
& \text { ماجستير ، كلية الزر اعة - جامعة بغداد. }
\end{aligned}
$$

Al-Dulaimy, H.H.A; A.M. Goma \&M.B. Al-Aswad (1985).Changes in a volatile nitrogen and free amino acids of goat meat stored by cooling and freezing.

Iraqi J. Agric. Sci. (ZANCO) 3 (3) : 51- 65.

Al-Suraify, Abdulkhalik T.A. and M.B. Al-Aswad (1986).Chemical and bacteri-

ological changes during aging of meat from old sheep. Iraqi J.of Agric.Sci. (ZANCO) 4 (4): $37-45$.

Anglemier , A . F ; A .A . El-Badawi and R .F. Lain (1964) . Effect of irradiation and pre - irradiation treatments on beef muscle proteins. J. Food Sci., 29:837 842

Chen,T.C.and C. Waimaleongora (1981).Effects of $\mathrm{pH}$ on TBA values of ground raw poultry meat . J. Food Sci., 46:1946- 1947.

Egan, H.;R. Kirk and R. Sawger(1988).Pearsons Chemical Analysis of Foods- $8^{\text {th }}$ ed , longman Scientific and Technical $591 \mathrm{pp}$.

Mountney, G.J. (1976). Poultry Products Technology. Second edition, Westport Connecticut the AVI Publishing Co. Inc.

Nam, H. J.;C.K.Park;D.G.Kimsong;Y.H. Moon and I.C. Jung (2000 ). Effect of freezing and refreezing treatments on chicken meat quality. J. Food Sci. (Korea) 18 - 29. Pearson , D. ( 1976 ) .The Chemical Analysis of Foods .Chemical Publishing Co., INC., New York, USA .

SAS (1996).Statistical Analysis System, SAS User Guide; Statistics .SAS INC., Larry, N.C.

Shehata, M.I.;M.A. Hussein;A.A.Al-Badawi and A.T.Habib(1970).Post mortem treatments of beef.111-Cold and freeze storge.Assuit J.Agric.Sci.1:87-94.

Woloszyn, J.;J.Ksiazkiewiez;A.Orkusz;T.J.Shrabka-Blotnicka \&T.Kisiel (2002). Preliminary evaluation of chemical composition of ducks Muscles from two polish flocks, $48^{\text {th }}$ I. cost $\left(48^{\text {th }}\right.$ International congress conservative of meat science and technology) FAO.Rome,25-30.August 2002-Vol. 1:374-375. 